\title{
DEVELOPMENT OF MICROMIRROR-BASED LASER LINE GENERATOR
}

\author{
By \\ Farzad Hossein Nia \\ BSc. Mechanical Engineering Azad University. Isfahan. 2012. \\ MSc. Amirkabir University of Technology. Tehran. 2014.
}

\author{
A MRP \\ presented to Ryerson University \\ in partial fulfillment of the \\ requirements for the degree of \\ Master of Engineering \\ in the Program of \\ Mechanical and Industrial Engineering
}

Toronto, Ontario, Canada, 2017

CFarzad Hossein Nia 2017 


\section{AUTHOR'S DECLARATION}

I hereby declare that I am the sole author of this MRP. This is a true copy of the MRP, including any required final revisions.

I authorize Ryerson University to lend this MRP to other institutions or individuals for the purpose of scholarly research.

I further authorize Ryerson University to reproduce this MRP by photocopying or by other means, in total or in part, at the request of other institutions or individuals for the purpose of scholarly research.

I understand that my MRP may be made electronically available to the public. 


\title{
Development of Micromirror-Based Laser Line Generator
}

\author{
Farzad Hossein Nia
}

Master of Engineering

Mechanical and Industrial Engineering, Ryerson University, 2017

\begin{abstract}
A laser line generator projects a straight line on an object and is widely used for alignment, identification, barcode reading, etc. Conventional laser line generators make use of cylindrical and Powell lenses that convert a laser beam spot into a line. Because of a fixed fan angle, however, the length and brightness of the generated line depends on the working distance between the laser module and object. In this project, using the optical system design software Zemax and engineering CAD software Solidworks, a micromirror based laser line generator is designed and fabricated. The performance of the fabricated micromirror based laser line generator is tested and compared with the performance of a conventional laser line generator. The results show that the length and brightness of image line can be kept constant by the designed generator.
\end{abstract}




\section{Contents}

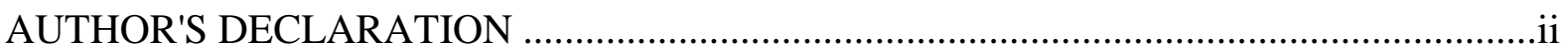

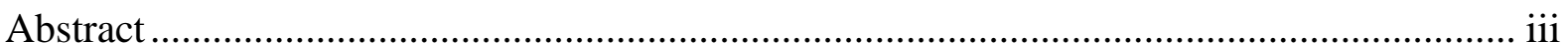

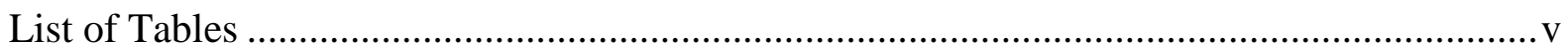

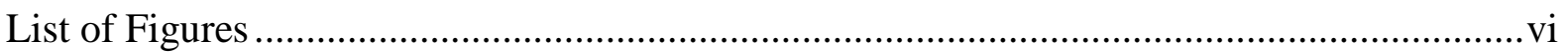

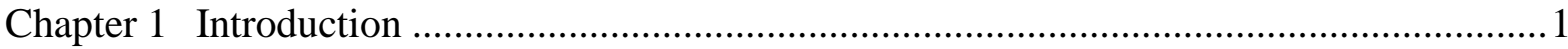

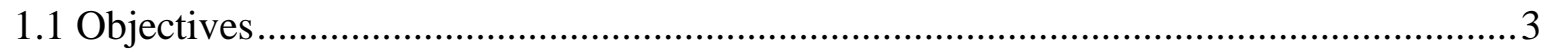

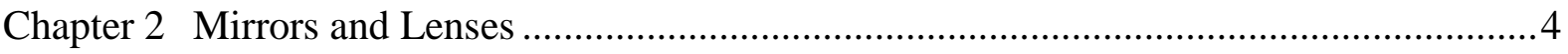

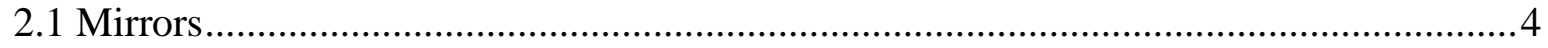

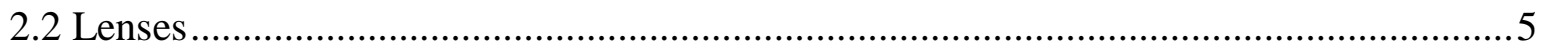

Chapter 3 Micromirror-Based Laser Line Generator ....................................................... 9

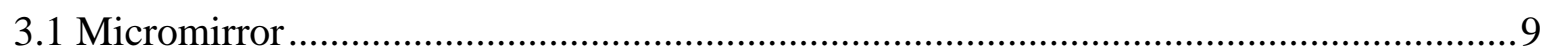

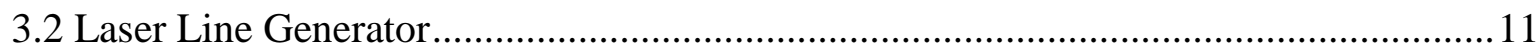

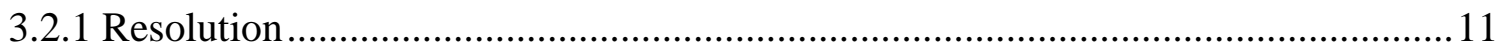

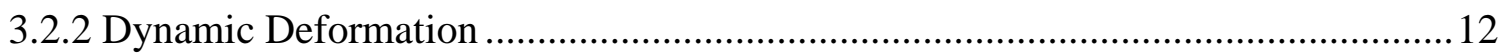

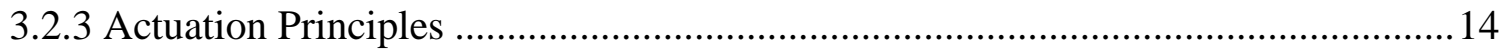

3.2.4 Optical Design Laser Line Generator ................................................................ 18

3.3 Advantages of Micromirror Laser Line Generator ..................................................20

3.4 Applications of Micromirror Laser Line Generator .................................................. 21

Chapter 4 Designing the Laser Line Generator by Zemax...............................................26

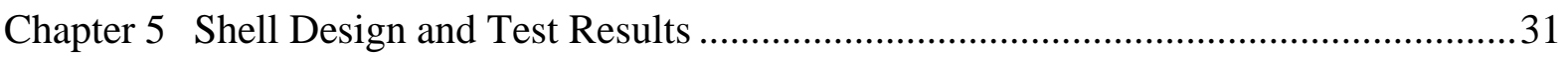

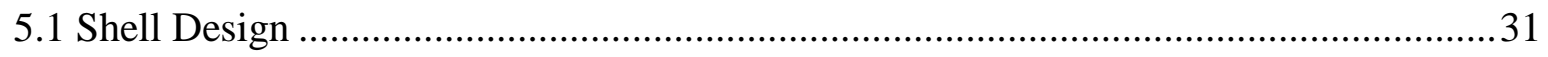

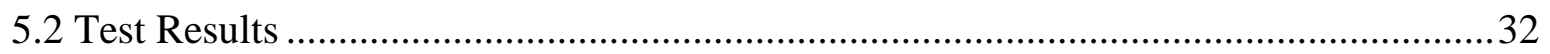

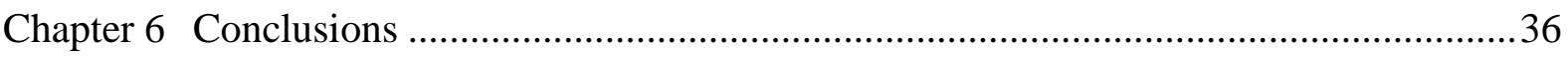

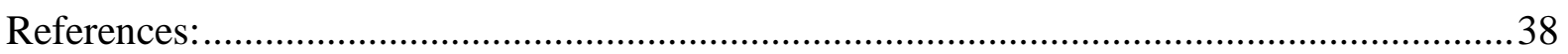




\section{List of Tables}

Table 3-1. Comparison of properties of actuation principles [26] ...................................... 15

Table 3-2.Comparison of linear imaging and laser scanning technology [39].......................23

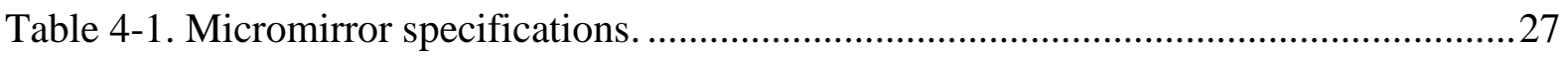

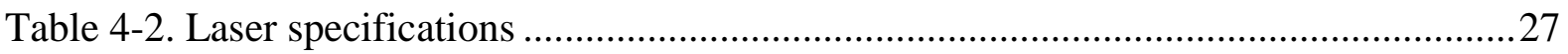

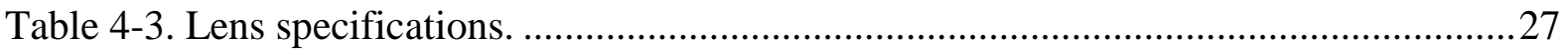

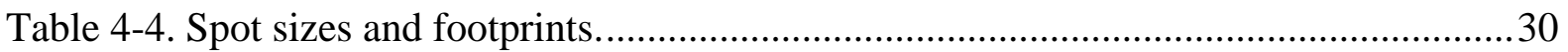




\section{List of Figures}

Figure 1-1. Laser lines generated by cylindrical and Powell lenses [2] ................................

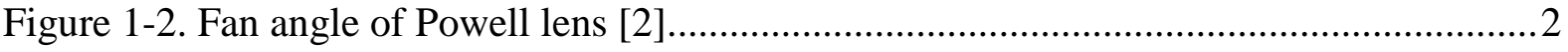

Figure 2-1. Parallel rays and their reflections [4].............................................................

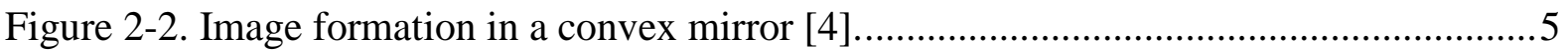

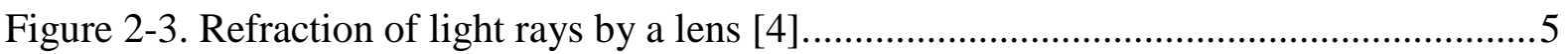

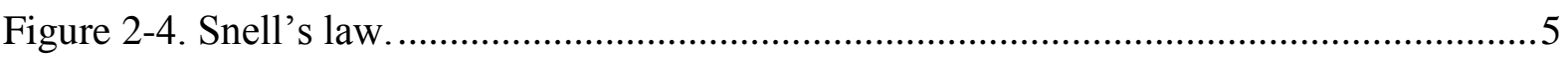

Figure 2-5. Different kinds of thin lenses [4] .................................................................6

Figure 2-6. The refraction of light in thin lenses [4]........................................................

Figure 2-7. Ray tracing through two lenses [4] ............................................................

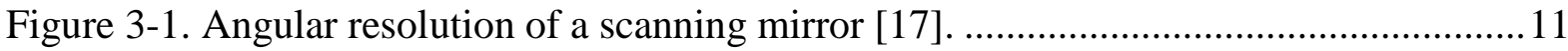

Figure 3-2. Mirror deformation during actuation [17]...................................................... 12

Figure 3-3.Maximum allowable actuating angle as a function of mirror frequency and

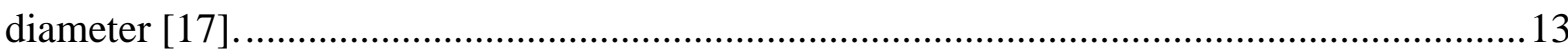

Figure 3-4. Parallel- plate (Top) and vertical combdrive (Bottom) electrostatic mirrors [28].

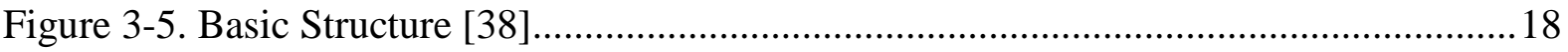

Figure 3-6. Angle amplification of a concave lens [38] ..................................................... 19

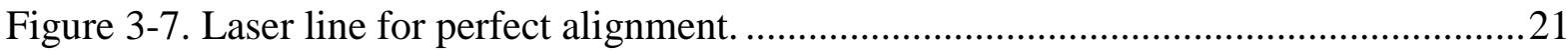

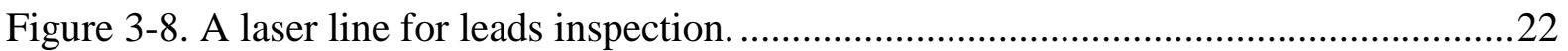

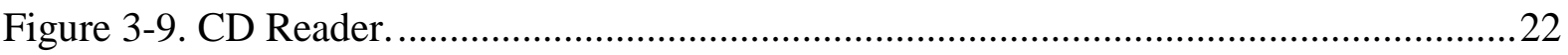

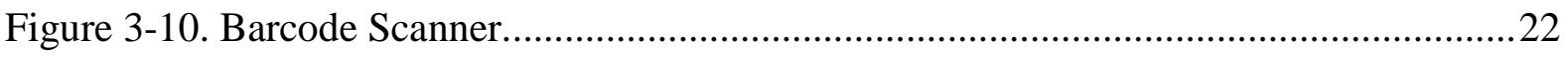

Figure 3-11. A typical structure of a laser printer [40] .................................................... 24

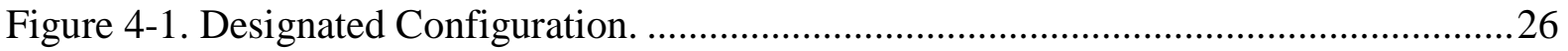

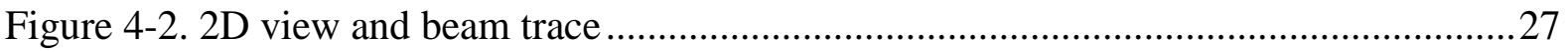

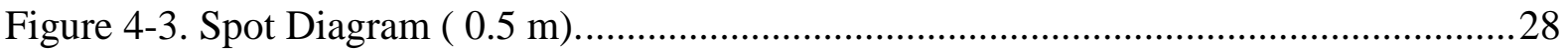

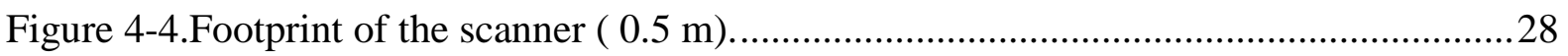

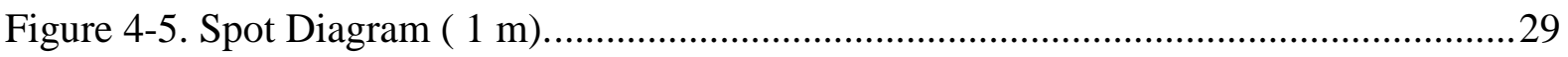

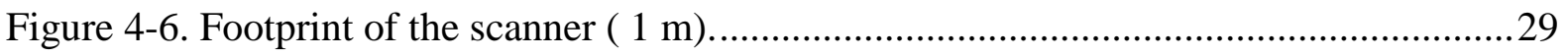

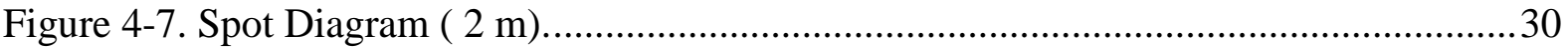

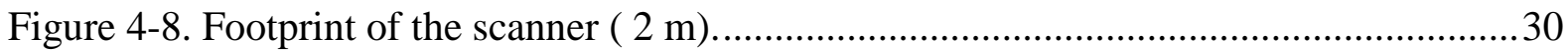

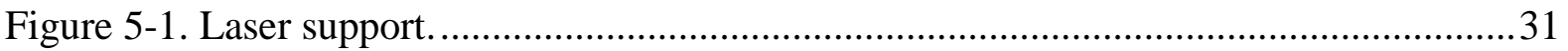




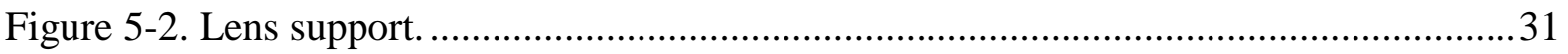

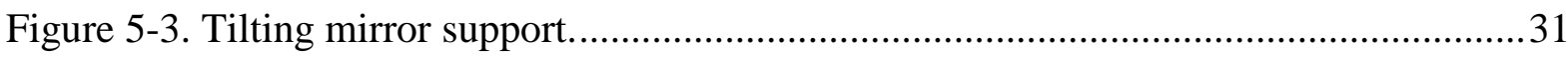

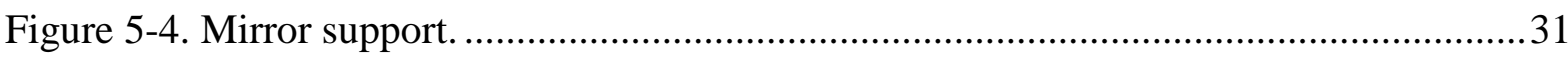

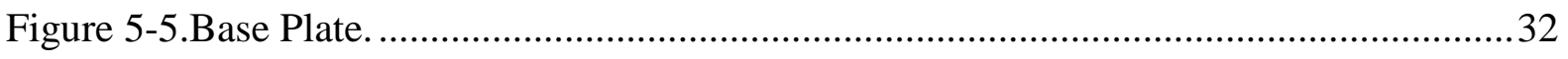

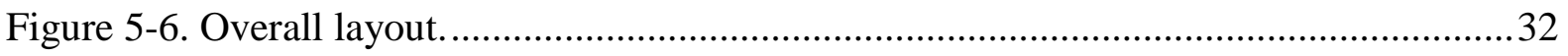

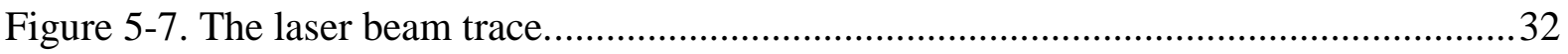

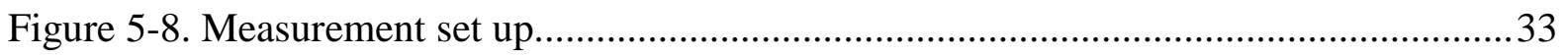

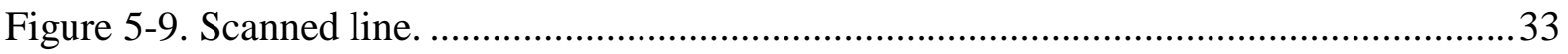

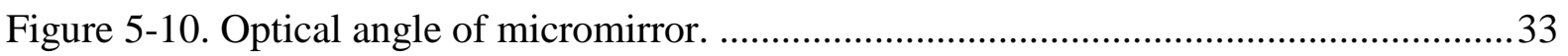

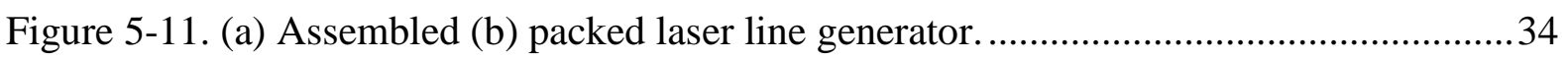

Figure 5-12.Brightness comparison of micromirror based laser line generator and conventional laser line generator at distances (a) $5 \mathrm{~m}$, (b) $1 \mathrm{~m}$, (c) $2 \mathrm{~m}$, (d) $3 \mathrm{~m}$. .35 


\section{Chapter 1 Introduction}

The term "laser" is an acronym for "Light Amplification by Stimulated Emission of Radiation". Lasers generate light beams that are monochromic, coherent and highly collimated. Lasers can be generally placed in four categories: gas lasers, semiconductor lasers, optical lasers and other types. Each category itself consists of many types with different wave lengths and applications. For example, a laser diode which is a kind of semiconductor laser, has applications in fiber optic communication, laser scanning, and laser printing.

Applications of laser are so numerous that is very difficult to list all of them. Scanning is one of the important applications of laser which has been widely used in pattern generation and imaging. Laser line generator is a one-dimensional scanner that can be fabricated with different technologies. Conventional laser line generators usually make use of two types of lenses: cylindrical lens and Powell lens. One kind of cylindrical lens, namely plano-convex type, can produce a thin line on the image plane. The linear spot generated by a cylindrical lens, however, has a Gaussian intensity distribution [1].

Powell lens, which has the shape of a circular prism with a curved roof line, can increase the uniformity of the linear spot [2]. Figure 1-1 shows the laser lines generated by Powell and cylindrical lenses.

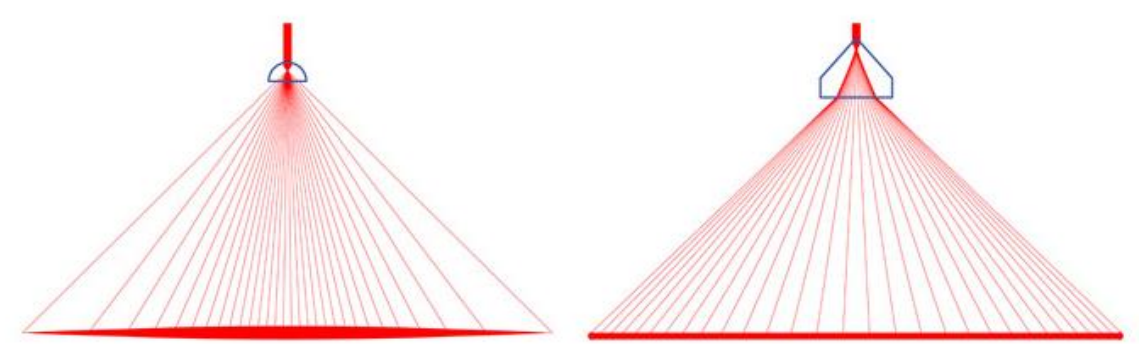

Figure 1-1. Laser lines generated by cylindrical and Powell lenses [2]

The fan angle of Powell lens is proportional to the refractive index of the glass and inversely proportional to its roof steepness (Figure 1-2). The laser beam dimension determines the laser line thickness at a given distance. To change the fan angle of Powell lens, it should be replaced by another lens. 


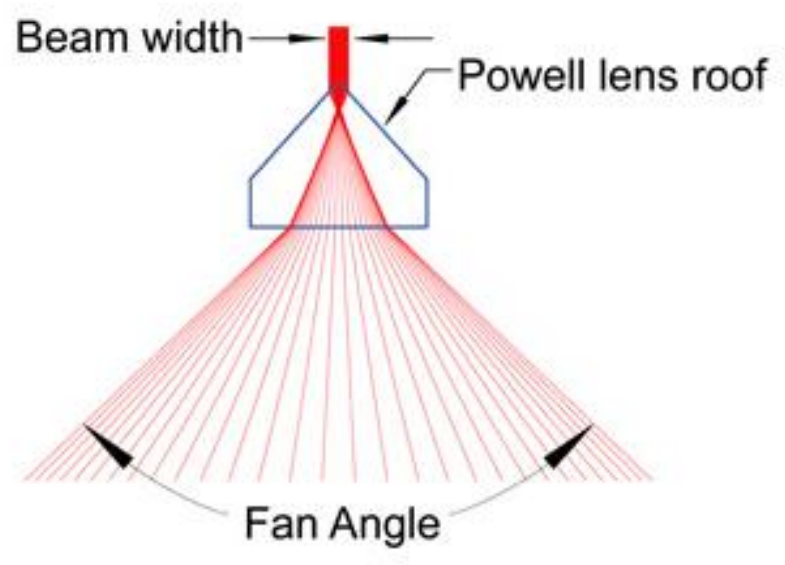

Figure 1-2. Fan angle of Powell lens [2]

Following to the invention of semi-conductor MEM micromirror, mirror scanners were also developed which offered many benefits as compared with conventional scanners. These benefits mainly stem from their small size, low weight, low power and high scan rate.

The first scanning silicon mirror was introduced in 1980[3]. Since then, micromechanical systems (MEMS) have been used for light scanning in a wide range of applications. At first MEMS scanners had their main applications in imaging such as barcode reading and finger print sensing. At the present time, micromirror scanners have many applications such as optical coherence tomography (OCT), spectroscopy, printing and light detection and ranging (LIDAR).

Two general areas for the applications of micromirror scanners are imaging and displaying. Comparing these areas, the demand for displaying applications is higher because imaging applications are limited by optics to generate small spot size with low signal levels.

The aim of this project is to design and fabricate a micromirror- based laser line generator (scanner) and compare its performance with the performance of a conventional laser line generator. Towards this goal, one has to consider the micromirror specifications and the performance principles of a micromirror based laser line generator Appropriate engineering softwares should then be used to design and fabricate the scanner. 


\subsection{Objectives}

The objectives of this project are the followings:

(1) Review of micromirror specifications and operation principles of micromirror based laser line generator.

(2) Designing a micromirror based laser line generator without the shell.

(3) Using a CAD software to design a case for all parts and fabricate the scanner.

(4) Testing the performance of the packed laser line generator and comparing it with the performance of a conventional laser line generator.

Mirror and lens are the main parts of a micromirror-based laser line generator. Chapter 2 is devoted to a brief review of geometric optics as related to these devices. Chapter 3 describes the micromirror specifications and the performance principles of micromirrorbased laser line generator. In Chapter 4, the software Zemax is used to design the scanner by selecting the laser, micromirror and the required optical lenses. This software is also used for analyzing the overall performance of the designed scanner. In Chapter 5, a shell is designed to hold all mechanical, optical and electrical parts of the scanner. To do this, the engineering software Solidwork is used to design the overall layout. The laser line generator is then assembled, packed and tested. These tests include the performance specifications such as resonant angle of the micromirror and the length of scanned line. Finally, some conclusions are drawn regarding the designed laser line generator in Chapter 6. 


\section{Chapter 2 Mirrors and Lenses}

Mirror and lens play important roles in forming a line image in a laser line generator. Hence, a brief description of these elements can be helpful in better understanding of the operation of laser line generator.

\subsection{Mirrors}

Two kinds of mirrors may be used in a laser line generator; plane mirror and spherical mirror. The object image with mirrors can be found by the law of reflection and ray tracing. The law of reflection says that a light ray is reflected from a mirror with the same angle as it approaches the mirror.

The main function of mirrors in a laser line generators, are to change or periodically divert the direction of a laser light. Figure 2-1 shows how parallel rays are deflected from two kinds of spherical mirrors; concave mirror and convex mirror. Parallel rays come from a very distant source or from collimated laser beam.

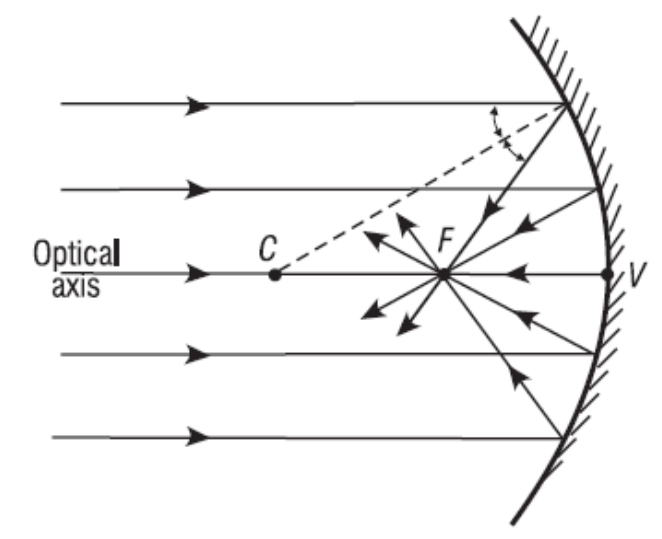

(a) Concave mirror

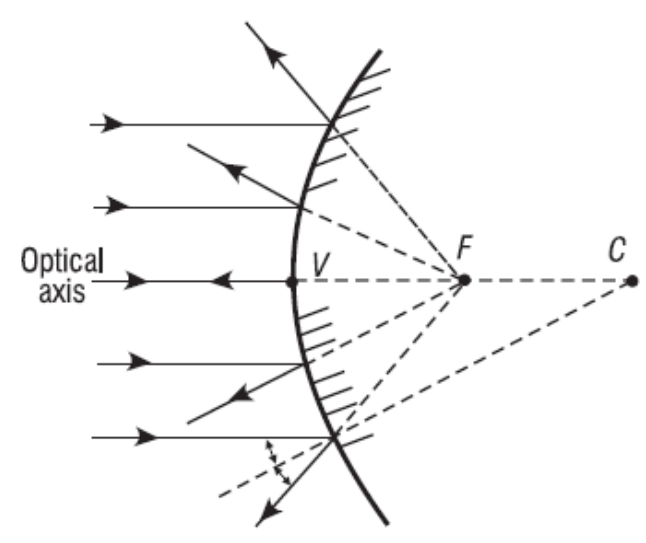

(b) Convex mirror

Figure 2-1. Parallel rays and their reflections [4].

Figure 2-2 illustrates how a virtual image is formed in a convex mirror. Convex mirrors are used, for example, in the driver side of automobiles. 


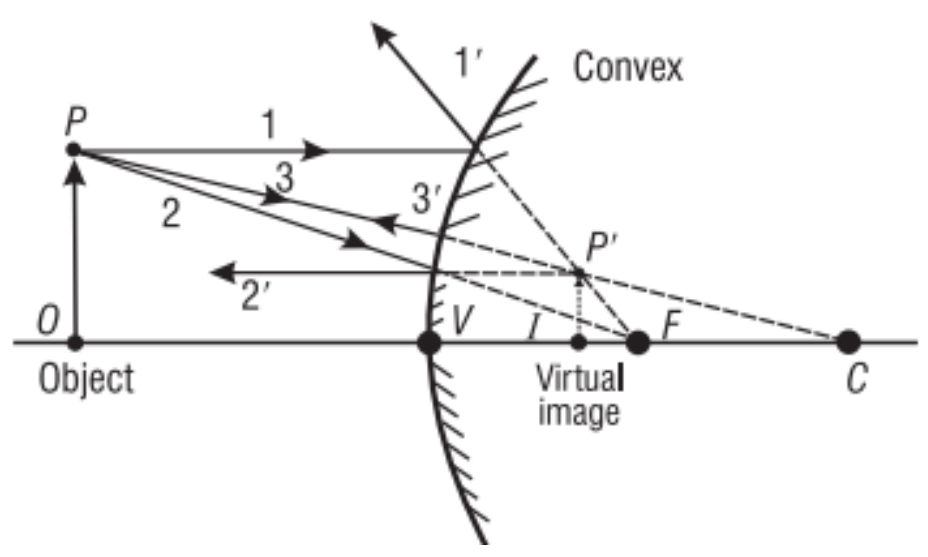

Figure 2-2. Image formation in a convex mirror [4].

\subsection{Lenses}

Lenses are the main parts of many optical devices such as cameras, microscopes and telescopes. A lens consists of a transparent refracting part with different shapes. When a ray hits the front surface, it refracts and propagates through the lens. By reaching to the back surface, it again refracts [Figure 2-3].

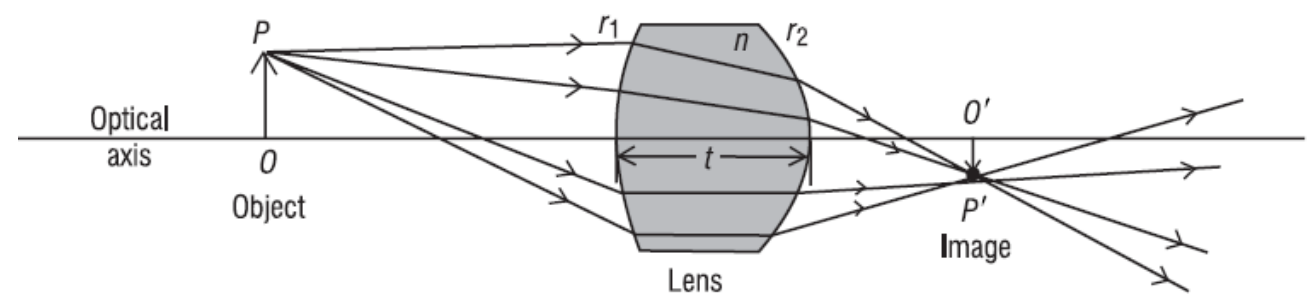

Figure 2-3. Refraction of light rays by a lens [4].

The image properties of lenses are determined by Snell's law which is summarized in Figure 2-4. Basically, lenses form images with visible light but can be also used for ultraviolet and infrared light.

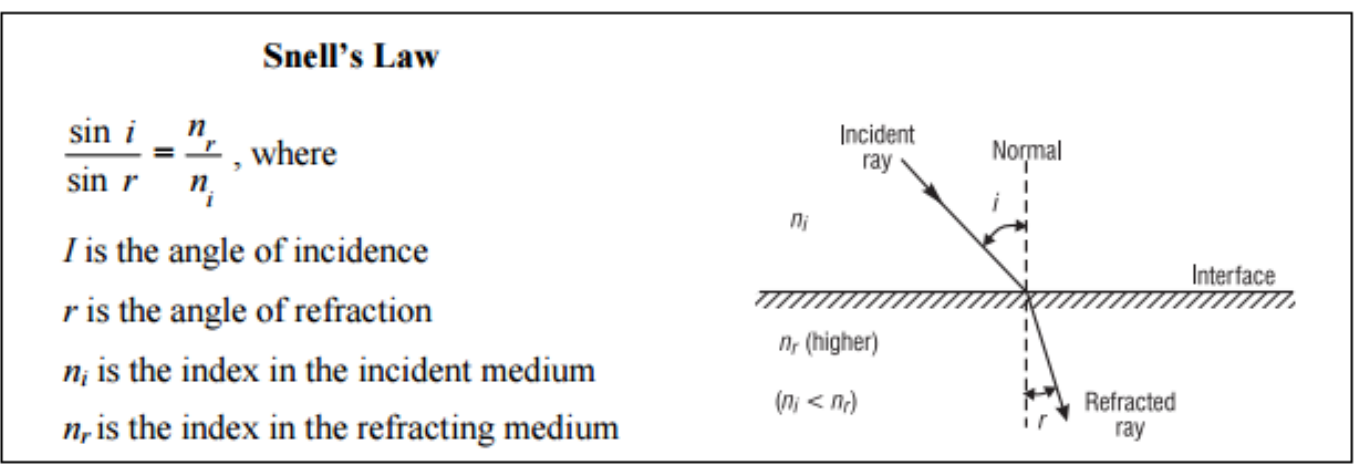

Figure 2-4. Snell's law [4]. 
If the thickness of lens is small compared with the radius of curvature of its surface, it is classified as a "thin" lens. Since ray-tracing can be easily done for thin lens, they are often used in the optical applications. Ray tracing is complicated for thick lenses; for which computer programs have been developed.

Thin lenses may have several shapes which are shown in Figure 2-5. When a thin lens is used in an optical system, it is assumed that the rays only refract at the front and rear faces and there is no translation through the lens medium. The first three lenses in Figure 2-5 are converging or positive lenses and the last three lenses are diverging or negative lenses.

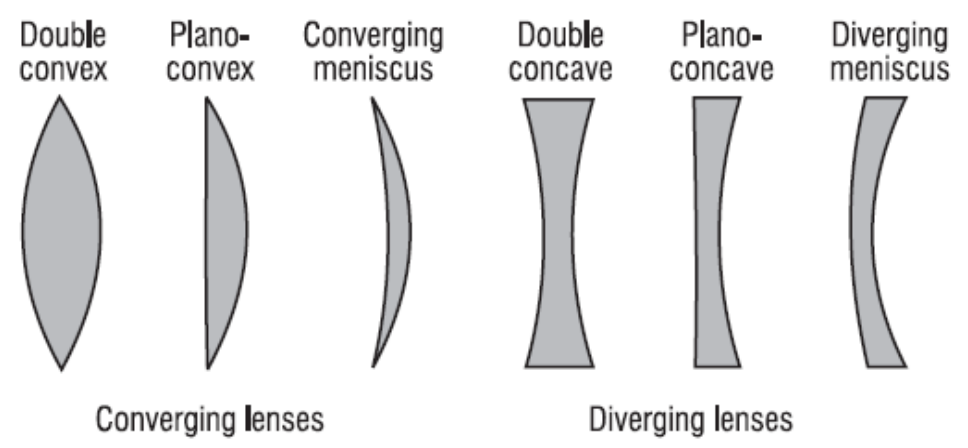

Figure 2-5. Different kinds of thin lenses [4].

A mirror has only one focal point and the light always remains in one side of the mirror. But a thin lens has two symmetrical focal points and the light can approach the lens from both sides. Figure 2-6 shows how the focal points may affect the light rays in positive and negative lens. 


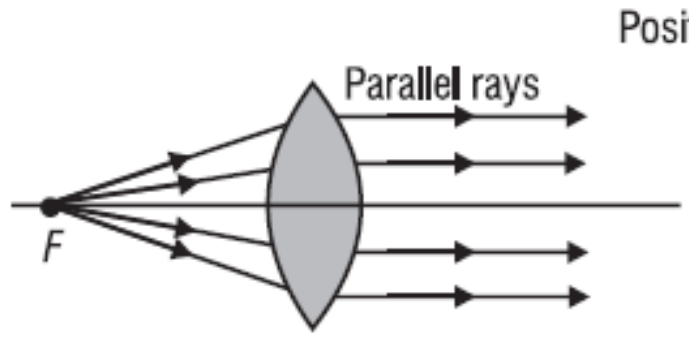

Left focal point

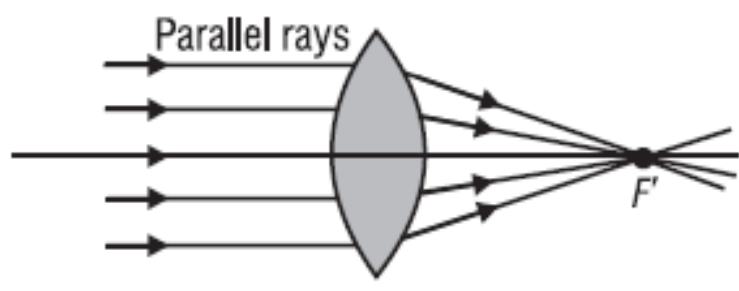

Right focal point

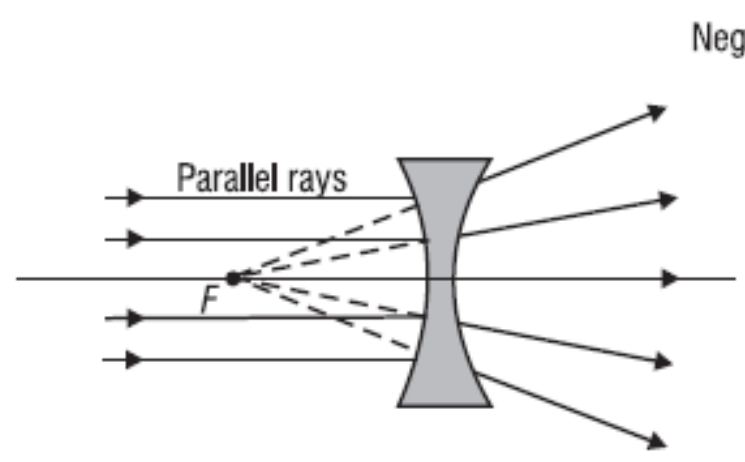

Left focal point

Negative lens

Figure 2-6. The refraction of light in thin lenses [4].

Figure 2.7 shows the ray tracing in a train of two lenses; a positive lens followed by a negative lens. In this figure, $\mathrm{F}$ stands for the focal length, $\mathrm{V}$ for the vertex of lens, $\mathrm{RO}$ for real object, RI for the real image and VI for virtual image.

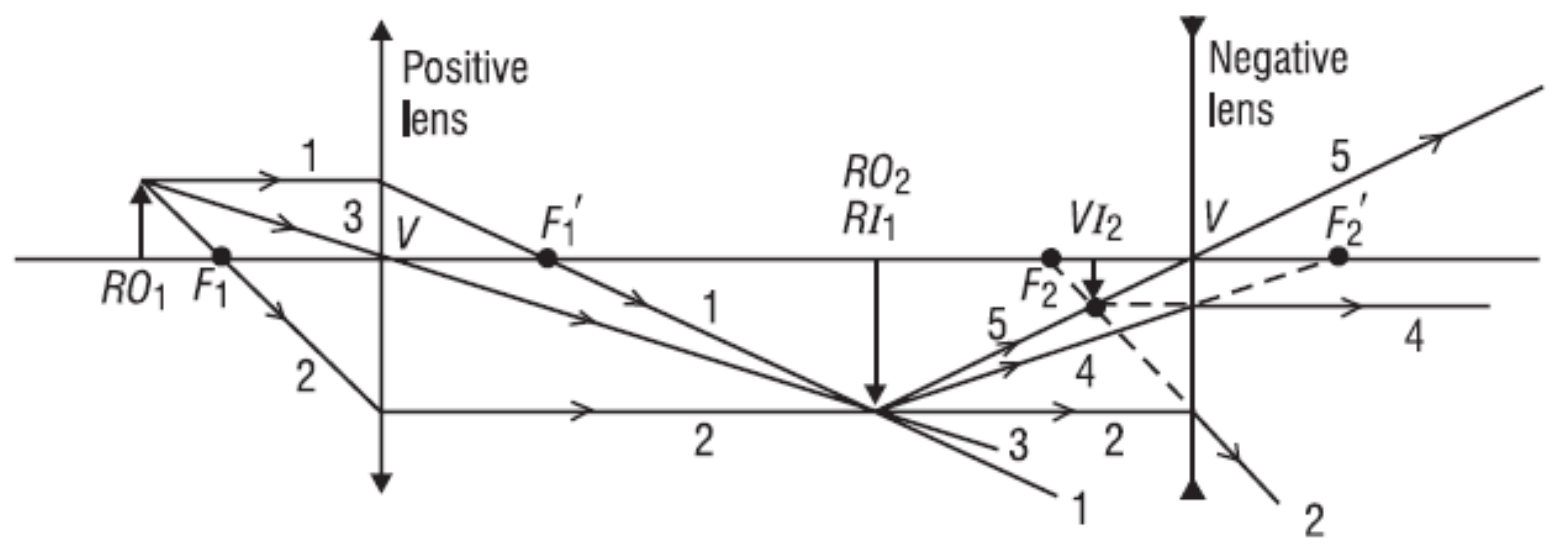

Figure 2-7. Ray tracing through two lenses [4].

An important parameter of a lens is called "f-number". The $f_{\text {number }}$ is also referred to as f/stop. It is defined as 


$$
f_{\text {number }}=\frac{f}{D}
$$

Where $\mathrm{f}$ is the focal length and $\mathrm{D}$ is the diameter of the lens. " $f_{\text {number }}$ " indicates the light gathering power or equivalently the brightness of a lens. In fact, it is shown that the image brightness of a lens is inversely proportional to the square of the " $f_{\text {number }}$ ". In summary, by increasing the diameter of a lens or selecting a lens with shorter focal length, the brightness of the image can be increased. 


\section{Chapter 3 Micromirror-Based Laser Line Generator}

Micromirrors are perhaps the first micro- optical devices fabricated by the microelectromechanical systems (MEMS) which were used for a wide range of applications in areas like optical communications and laser imaging and displays.

Micromirror-based laser line generator, which is a one dimensional scanner, has a large number of applications ranging from barcode reader to laser printer and medical tools. In this chapter, following a general description of micromirror specifications, the performance principles of a micromirror laser line generator and its design parameters are presented. Benefits and applications of laser line scanner are also mentioned.

\subsection{Micromirror}

MEMs micromirrors are the best candidates to replace macromirrors if miniaturization, lightweight, low energy consumption and reduced cost are required. To satisfy such requirements, many type of MEMs micromirrors, from small $(\sim 200 \mu \mathrm{m})$ micromirrors in large arrays to the very large (several to tens of $\mathrm{mm}$ ) scanners in high resolution imaging systems have been developed.

Micro scanning mirrors have been developed with different fabrication technologies (bulk and surface micromachining,etc.)[5], different materials (crystalline silicon[6], poly silicon [7], aluminum[8], silicon nitride[9], etc.), and different actuations(electrostatic[10], electromagnetic[11], piezoelectric[12], electrothermal[13]). SOI micromirror that is used in this project is a kind of micromirror which is fabricated by SOIMUMPs process [14]. MUMPs, or Multi-User MEMs Processes, provides MEMS fabrication procedure to industry worldwide. This is a silicon- on- insulator micromachining process. In this process, siliconon- insulator (SOI) wafer is used as the starting substrate.

The main problem for using a micromirror in a scanner is how to actuate it for the required angular movement. There are different actuating methods which will be reviewed in the next section. 
Micromirrors can operate in two major modes [15]:

(1) Point - to - point mode (quasi- static mode): in this mode, a steady - state analog actuation voltage results in a steady- state angle of rotation of the micromirrors. Therefore, mirror can hold a DC position, or move in uniform velocity, or perform vector graphics.

(2) Resonant mode: in this mode, low actuating voltage of a frequency near resonance results in a large beam angle. Resonant frequency depends on actuator type and micromirror type and diameter.

It may seem that static operation is more convenient because a linear scanning can be obtained with a constant beam velocity. But to have a linear scanning at high frequency, there is a need for a strong actuation, which in turn introduces deformation in the micromirror.

A resonant micromirror, on the other hand, consumes less power with a larger scanning angle. In fact, resonant micromirrors can replace the static actuated micromirrors in most applications.

There are some parameters that should be considered in using micromirrors: radius of curvature, surface roughness, reflectivity and damage threshold.

Radius of curvature which is given by the manufacturers directly affects the scanner specifications and lenses.

Laser reflection from a non-smooth surface causes interference in the beam coherence. Laser speckle is the name which is given to this interference.

Reflectivity of micromirror depends on the beam angle of incidence and wavelength and its coating. Aluminum (AL) and gold (Au) are two common coatings. AL coating performs well at all wave lengths, while Au coating can compensate the surface heat. Micromirrors with different coatings can have reflection coefficient of $80 \%$ up to $99 \%$.

Damage threshold of micromirror depends on specific coating, wave length and mirror size, and is given in $\frac{j}{\mathrm{Cm}^{2}}$. A typical value for the damage threshold is $2 \frac{j}{\mathrm{Cm}^{2}}$. One can increase the damage threshold by selecting a larger mirror, but it results in lower speed because of increased inertia. In fact, the inertia of circular mirror is proportional to the fourth power of its radius. 
Micro mirrors can be employed in two forms: integrated and bonded. Integrated mirrors are small (typically 2mm) and thin (about 40 micrometer). Bonded mirrors are fabricated separate from MEMs device/actuator.

\subsection{Laser Line Generator}

A micromirror laser line generator is usually constructed from a laser source, a tunable mirror and some lenses. It illuminates an object with a moving spot which is seen by an observer like a continuous line.

The performance of a laser line generator depends on working frequency, scan angle, mirror size, mirror flatness and operation mode (i.e. resonant or non resonant scanning)[16]. In fact the mode of operation affects the image quality, while the first four factors determine the image size and scanner resolution.

The main problem in designing a resonant scanner is to avoid image distortion by keeping the mirror flat; while the most difficult problem in a non-resonant scanner is its linearity.

Resolution and dynamic deformation are two important characteristics for micromirror based line generator which are discussed in the following.

\subsubsection{Resolution}

The resolution of a scanner is an important factor which characterizes its performance. The scanner resolution is defined as the number if elements (pixels) that can be discriminated across the image length, i.e. the number of spots per line (Figure 3-1).
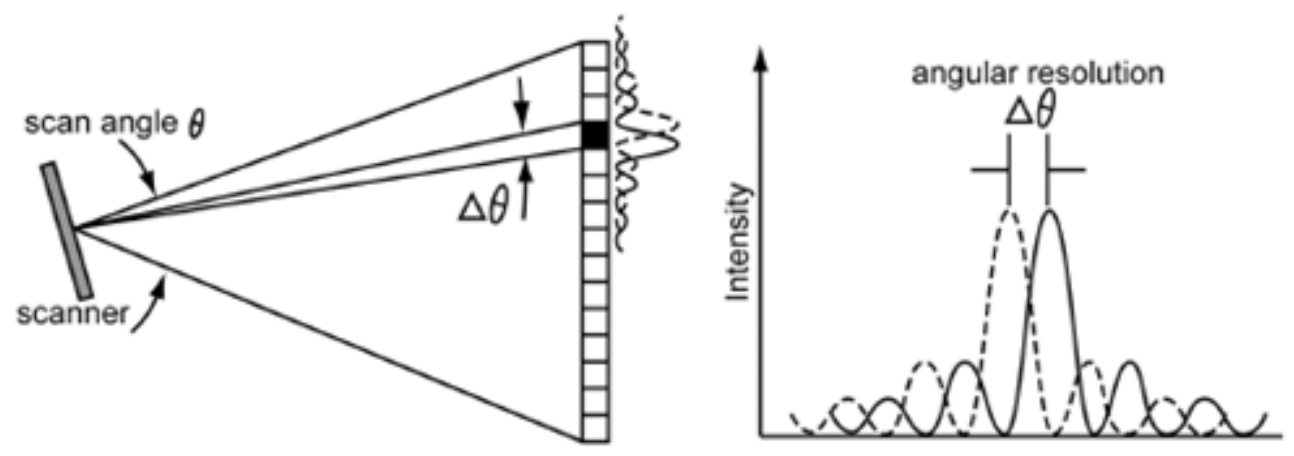

Figure 3-1. Angular resolution of a scanning mirror [17].

There are two main parameters that determine the number of resolvable image spots, namely, mirror aperture and scan angle. For small scan angle, the resolution is given by 


$$
N=\frac{\theta \mathrm{D}}{a \lambda}
$$

Where $\theta$ is the maximum scanning angle (which is twice the maximum deflection angle of the mirror, $\left.2 \theta_{m}\right), \mathrm{D}$ is the diameter of the mirror, $\lambda$ is the wavelength of the laser and " $a$ " is a parameter which depends on the mirror shape. The values of " $a$ " are given for different shapes in $[18,19]$. Typically, ' $a$ ' has a value between 0.75 and 2 . Note that $\mathrm{N}$ is proportional to $(\theta . D)$.

\subsubsection{Dynamic Deformation}

The mirror deformation is the most difficult problem in designing a high frequency resonant scanners. In fact, mechanical deflection causes mirror bending that results in mirror distortion ( Figure 3-2 ). Dynamic deformation, $\delta$, is given by formula (3-2) [20].

$$
\delta_{\max }=0.217 \frac{\rho f^{2} D^{5} \theta_{m e c h}}{E t^{2}{ }_{m}}
$$

where $\theta_{m}$ is the maximum zero-to peak angle of tilting mirror, $\rho$ is the density of mirror material, $\mathrm{E}$ is the modulus of elasticity and $t_{m}$ is the mirror thickness. To avoid image distortion, $\delta$ should be less than $\lambda / 8$ where $\lambda$ is the shortest system wavelength. As it is depicted from Equations (3-1) and (3-2), designing of scanner involves a trade off between different parameters of scanner [21]. For example, increasing the scanner resolution by selecting larger micrimirror results in more mirror deformation..

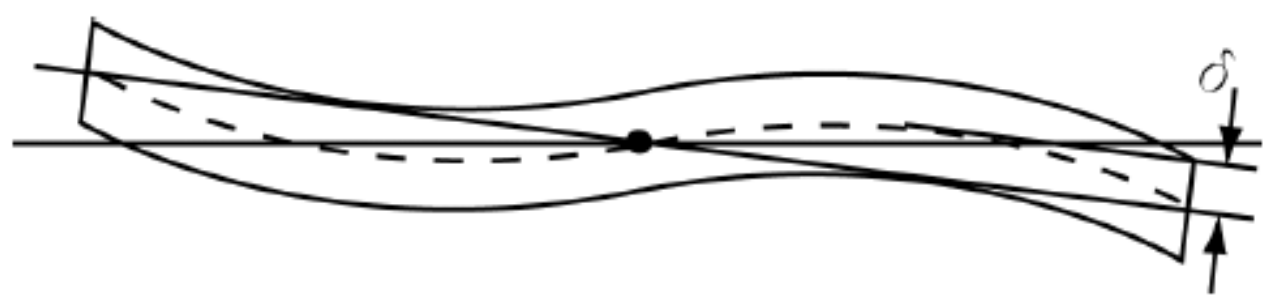

Figure 3-2. Mirror deformation during actuation [17].

The maximum allowable actuating angle of a micromirror is limited by its deformation which is in turn a function of the mirror actuating frequency and diameter. Figure 3-3 shows the maximum allowable actuating angle as a function of mirror frequency and diameter for deformation less than $\lambda / 10$. 
A mirror scanner operating in mechanical resonance oscillates harmonically. Hence, the speed of scan is not constant across the scan pattern [22, 23].

The angle $\theta$ of the micromirror at resonance is

$$
\theta=\theta_{m} \sin \left(w_{r} t\right)
$$

where $\theta$ is the mirror angle, $\theta_{\mathrm{m}}$ is its maximum deflection angle and $\mathrm{w}_{\mathrm{r}}$ is the angular resonant frequency of the micromirror. The resonant frequency of micromirror is determined by the mechanical properties of the scanner. Specifically, the resonant frequency for a torsional micromirror is given by

$$
w_{r}=2 \pi f_{r}=\sqrt{\frac{K}{I}}
$$

where $\mathrm{I}$ is the mass moment of inertia $\left(\frac{1}{12 m L^{2}}\right.$ for square mirror), $\mathrm{k}$ is the spring constant, $\mathrm{m}$ is the mass and $\mathrm{L}$ is the diameter of the micromirror.
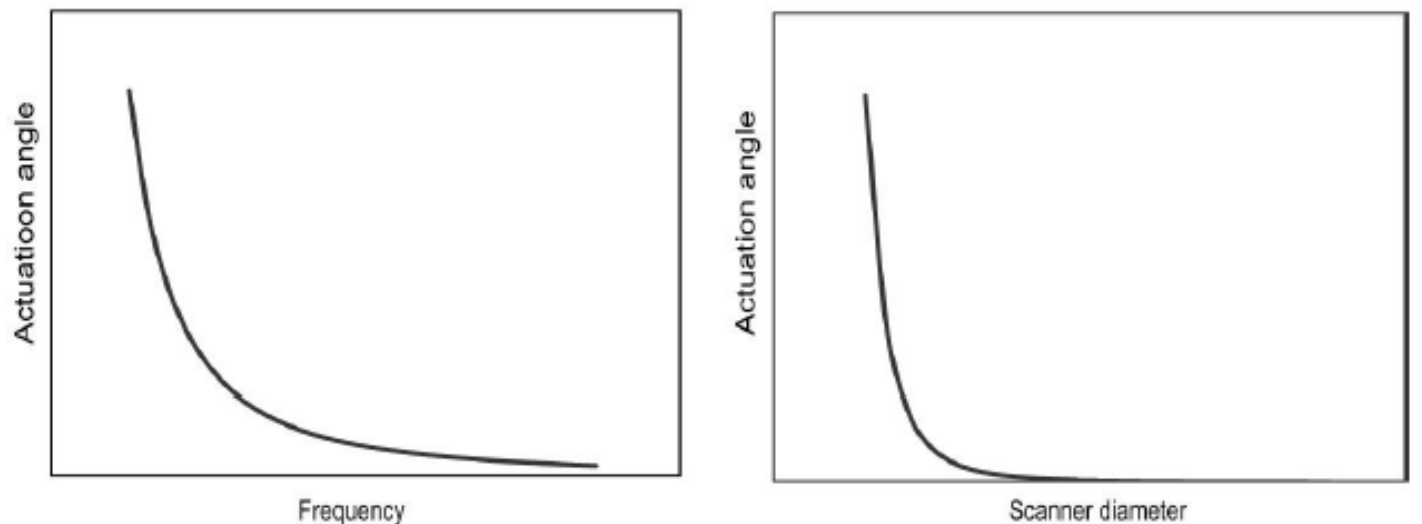

Figure 3-3.Maximum allowable actuating angle as a function of mirror frequency and diameter [17]. 
Using Equation 3-3, the angular-scan rate is given by

$$
\frac{\partial \theta}{\partial t}=w_{r} \cdot \theta_{m} \cdot \cos \left(w_{r} \cdot t\right)
$$

The linear displacement $\mathrm{X}$ in the image plane is given by

$$
X=s \cdot \tan \theta
$$

where $\mathrm{S}$ is the distance from mirror to the image plane. Therefore, the linear scan rate (velocity) of the laser beam is given by

$$
\frac{\partial X}{\partial t}=2 s\left(1+\tan ^{2} \theta\right) \cdot \frac{\partial \theta}{\partial t}
$$

Note that, the velocity of laser beam can be computed for any t or $\theta$ from above equations.

\subsubsection{Actuation Principles}

As stated previously, the main micromirror actuating methods are electrostatic actuation, electromagnetic actuation, piezoelectric actuation and electrothermal actuation. Electrothermal actuation is not suitable for micromirror laser line generators because of its long response times[24, 25]. If combined with a fast scanner, this method may be suitable for a $2 \mathrm{D}$ scanner.

Table 3-1 compares the properties of three more important methods [26].In this table, displacement is relevant to the scanning angle, force corresponds to the extent of mechanical force exerted to the mirror and linearity shows how linear the scanner behaves. The actuation methods are more described in the following.

Electrostatic Actuation: Electrostatic method was the first actuating procedure employed by MEMs scanners. It was due to its fabrication compatibility and integration with MEMs. Electrostatic actuation is still the predominant method used for MEMs scanners.

Torsional rotation of micromirror by electrostatic actuation is performed by two electrodes: fixed and movable electrodes. The basic principle is that a voltage is applied between the fixed and movable electrodes that make the mirror rotate about its axis until the electrostatic 
Table 3-1. Comparison of properties of actuation principles [26].

\begin{tabular}{|l|c|c|c|}
\multicolumn{1}{c}{ Category } & \multicolumn{3}{c|}{ Electrostatic } \\
\hline Preferred Type & Comb drive & Moving coil & PZT film \\
\hline Simple Fabrication & $\checkmark \checkmark \checkmark$ & $\checkmark \checkmark \checkmark$ & $\checkmark \checkmark$ \\
\hline Large Displacement & $\checkmark \checkmark$ & $\checkmark \checkmark \checkmark$ & $\checkmark$ \\
\hline High Force & $\checkmark$ & $\checkmark \checkmark$ & $\checkmark \checkmark \checkmark$ \\
\hline Low Power & $\checkmark \checkmark$ & $\checkmark$ & $\checkmark \checkmark \checkmark$ \\
\hline Low Voltage & $\checkmark$ & $\checkmark \checkmark \checkmark$ & $\checkmark \checkmark$ \\
\hline Compactness & $\checkmark \checkmark \checkmark$ & $\checkmark$ & $\checkmark \checkmark \checkmark$ \\
\hline Linearity & $\checkmark$ & $\checkmark \checkmark \checkmark$ & $\checkmark \checkmark$ \\
\hline
\end{tabular}

torque becomes equal to the opposing torque. The electrostatic torque depends on the capacitance of actuator, which in turn depends on the area of electrodes overlap and the distance (gap) between the electrodes.

There are two major configuration of electrostatic actuation for scanning micromirror: parallel-plate and vertical comb drive (Figure 3-4). Most of early micromirror based scanners made of parallel-plate actuation [27]. This method is still used but has a limitation in scan angle. In fact, to have large scan angle, the electrodes gap must be increased that results in applying a higher voltage.

As far as the fabrication is concerned, parallel-plate scanners can be fabricated by either surface or bulk micro machining processes, or their combination. The Multi-User MEMs process (MEMPs) [14] can be employed for the fabrication of surface micromachined scanners.

Vertical comb drive has a better performance as compared with parallel -plate actuator because the distance between the electrodes are fixed and scan angle is independent if gap size. As seen in Figure 3-4, there are two finger shaped electrodes in vertical comb drive. One static and one movable with fixed distance. 

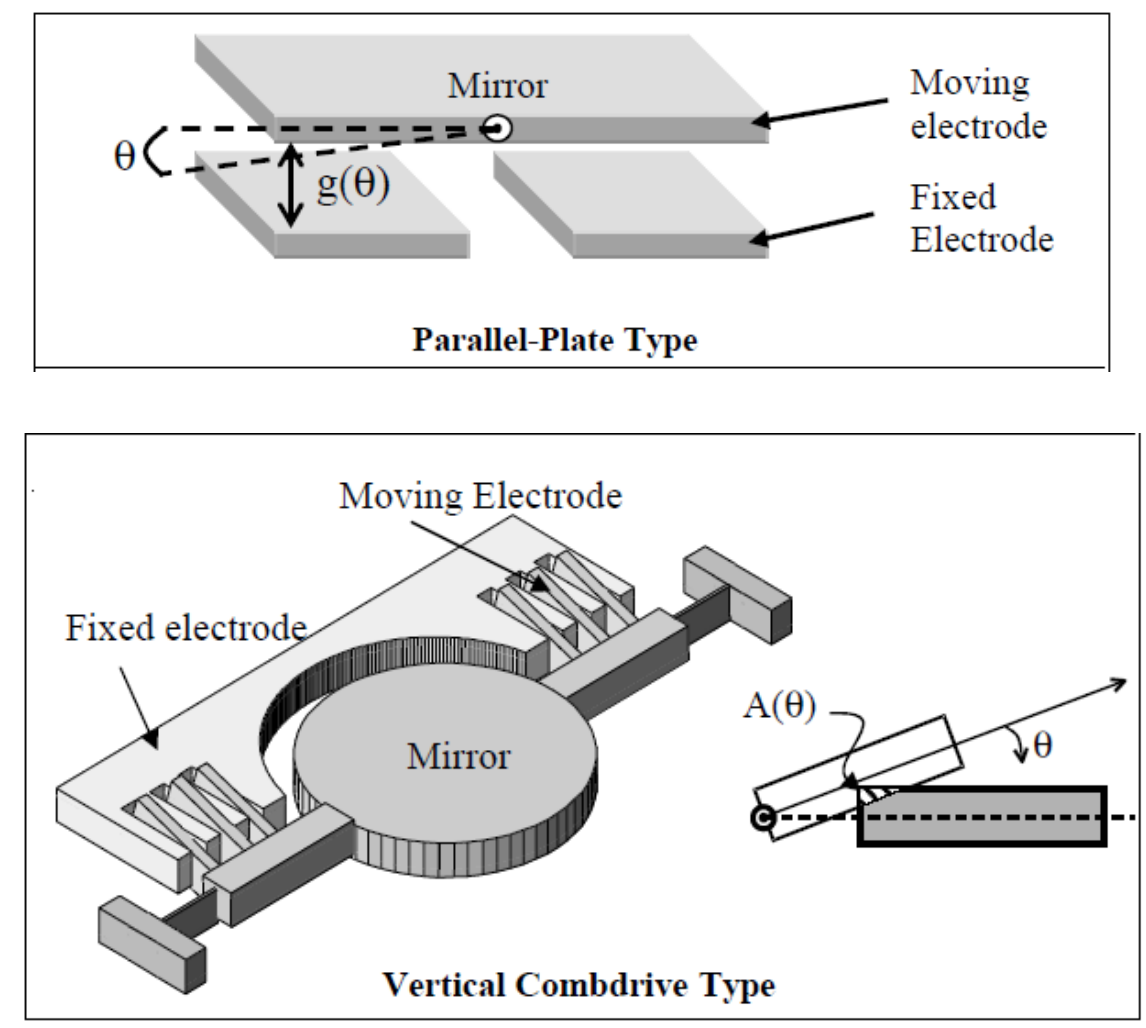

Figure 3-4. Parallel- plate (Top) and vertical combdrive (Bottom) electrostatic mirrors [28].

The vertical combdrive, however, is more employed in different applications. The first vertical combdrive was purposed by Selvakummar et al [29].

By incorporating micro mechanical hinges, electrostatic actuators can generate out of plane movement suitable for 3-dimensional motion. Electrostatic actuation can be employed in high rate scanning applications.

Generally, electrostatic actuation has the benefits of small size, stability, and can be fabricated by use of SOIMEMs. This method, however, requires high voltage and is very sensitive to exact fabrication.

Electromagnetic Actuation: Electromagnetic actuator may have two configurations: moving magnet and moving coil. The first type makes use of an external coil together with a magnet. It can be simply fabricated and there is no need for electrical contacts with the scanner. In the moving coil configuration, a coil is fabricated with scanner and an external magnet is used to produce a magnet field [30, 31]. Using silicon on magnetic actuator can give \pm 10 degrees angular deflection for fields of $0.07 \mathrm{~m} \mathrm{~T}$. Integrated or ultraminiaturized coils can be used for generating the required magnetic field. 
Electromagnetic actuation is more suitable for larger micromirrors [28]. The reason is that the magnetic torque is proportional to the permanent magnetic material. Moreover, magnetic actuation has a more linear response compared with electrostatic actuation and is best suited for linear slow scanners. In summary, electromagnetic actuator has a relatively large size but with low power consumption.

Piezoelectric Actuation: Since reliable and convenient methods were developed for fabrication of piezoelectric actuator, it has been accepted as a major actuation principle for MEMs scanners. Fabrication of piezoelectric actuators for MEMs scanners is performed by film decomposition of piezoelectric material on the scanner [32].

The working principle of piezoelectric actuator in MEMs is simple, piezoelectric film expands when it is actuated by a voltage. Lead ziroconate titanate (PZT) is the most common piezoelectric material used for MEMs scanner actuation. Piezoelectric actuation has a higher efficiency compared with electromagnetic actuation and works with lower voltage as compared with electrostatic actuation. Limited scan angle can be considered as a drawback for piezoelectric actuation principle.

All types of actuation methods can be used in resonant scanners. Electrostatic actuation has more applications in these scanners but it requires a relatively high voltage. Electromagnetic actuated scanners can provide high scan angles with relatively small voltages. Piezoelectric actuated scanners are the best among the high frequency resonant scanners $(>30 \mathrm{KHz})$.

As far as the reliability of micromirror is concerned, the stress applied to the actuator springs should be smaller than the yield stress of the mirror material. Also, the mirror geometry must be considered in designing the actuator springs. If a mirror is used at high frequency, some cracks may appear in the mirror as the result of applied stress. These cracks cause a frequency shift in the actuating frequency or even results in the mirror breakage. The formation of cracks can be detected by measuring the resonant frequency [33-35].

Based on the structure of actuation, the scanners can be classified as Direct and Indirect drives. In direct drive, the torque is directly applied to the mirror frame. In a scanner with the indirect drive, a resonant mode is coupled to mirror by the motion of a larger mass. This kind of mechanical coupling is more applicable to electromagnetic and piezoelectric actuations $[36,37]$. 
Comparing the two drive methods, direct drive is easier to design and has more applications. This method is best fitted to the scanners with piezoelectric actuation.

\subsubsection{Optical Design Laser Line Generator}

Figure 3-5 shows a simple yet effective configuration of a laser line generator. The positive lens is usually included in an adjustable laser module. Due to small diameter of micromirror, its fan angle is small (typically 5 to 15 degrees). This can be considered as a limiting factor because longer image line or wider field of view is usually required. Using a concave lens after micromirror can result in fan angle amplification and, consequently, longer image line. At the same time, it also keeps the ratio of beam diameter to image distance very small; i.e. small spot size at the image. The concave lens also compensates for the micromirror curvature.

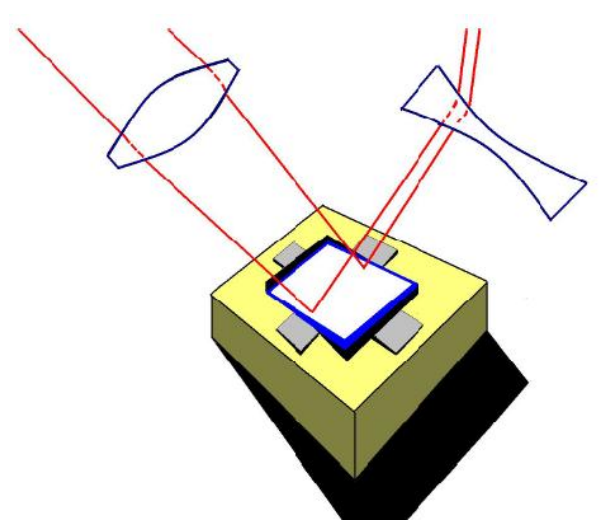

Figure 3-5. Basic Structure[38].

Figure 3-6 shows how the fan angle of a converging beam from micromirror is amplified by a double concave lens. In this Figure $\theta_{i}$ and $\theta_{0}$ are the angles of the main beam rays with respect to the optic axis. Two marginal rays together with their heights $\left(h_{0}, h_{1}, h_{2}\right)$ at the concave lens are also shown. $P$ is the distance between micromirror and lens, $f_{n}$ corresponds to focal point of the lens, and $l_{0}, l_{1}, l_{2}$ denote the distances from the intersection of the extensions of output rays with focal plane to the optic axis. Based on this figure,

$$
h_{0}+l_{0}=f_{n} \cdot \tan \theta_{0}
$$


which results in

$$
\tan \theta_{0}=\left(1+\frac{p}{f_{n}}\right) \tan \theta_{i}
$$

Given $P$ and $f_{n}$, the above equation can be used for the angle amplification factor of concave lens. Note that the spot diameter Ds at a distance $\mathrm{L}$ can be related to the angle $\Delta \theta=\theta_{2}-$ $\theta_{1}$ by the following relation:

$$
D_{s}=\Delta \theta \cdot L
$$

Where $\Delta \theta$ can be found from input beam diameter [38].

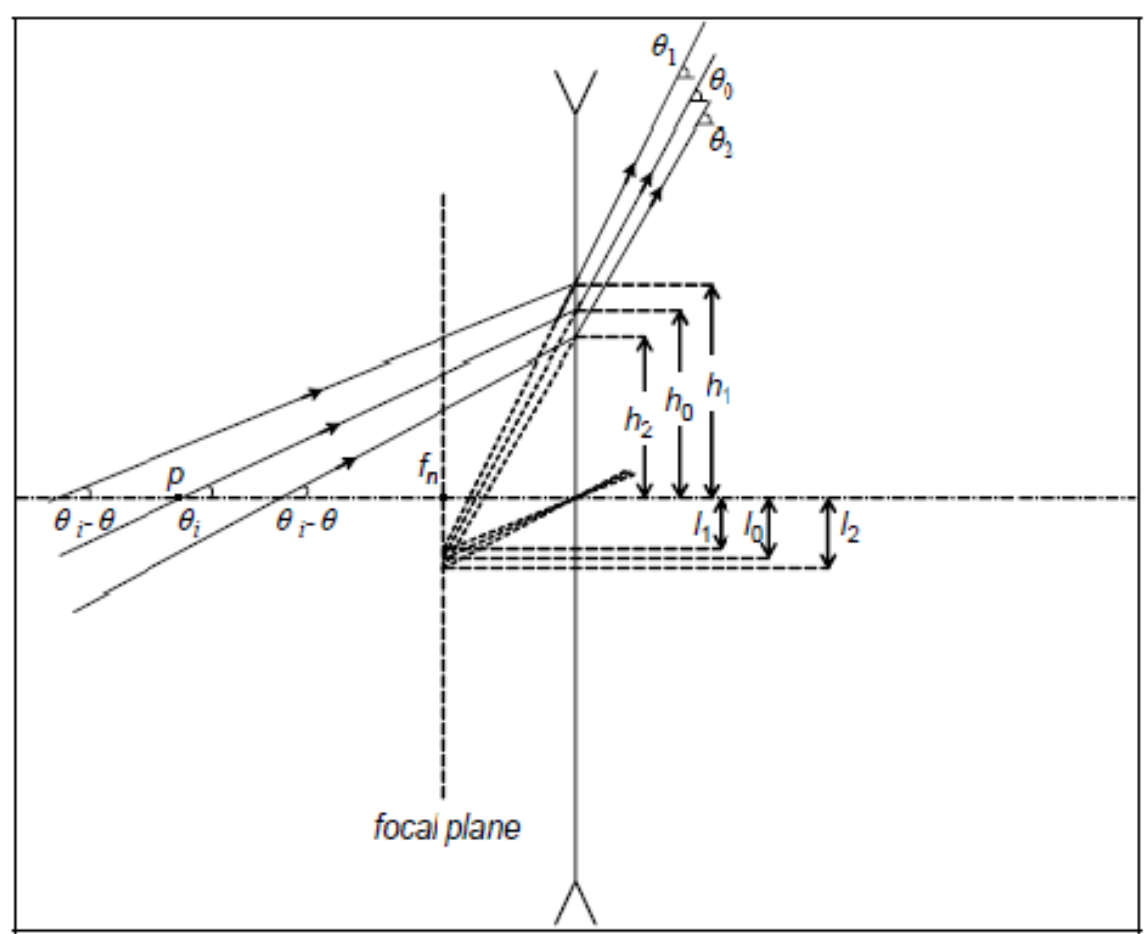

Figure 3-6. Angle amplification of a concave lens [38].

By moving the concave lens with respect to the micromirror, the optical fan angle can be changed without changing the number of spot point; as the resolution depends on the tilting angle of micromirror. It means that one can zoom on the image with a fix resolution. This property is one advantage of micromirror based laser line generator as compared with conventional one. 
Micro mirror laser line generator as a one- dimensional laser scanner has applications have been reported. The benefits of micro mirror laser line generator differ for each specific application. Hence, the general beneficial characteristics of mirror scanner are first mentioned and then its potential use for each application is discussed.

\subsection{Advantages of Micromirror Laser Line Generator} Small size:

Micromirror can be fabricated with small surface dimensions (e.g. $1.0 \mathrm{X} 1.0 \mathrm{~mm}$ ) and integrated with the required actuator circuit. This results in a small scanner module with a high structural resistance.

\section{High scanning rate:}

Can operate with a frequency in the range of $20 \mathrm{~Hz}$ up to more than $100 \mathrm{KHz}$. So, it can speed up some devices like laser printers.

\section{Low distortion:}

Can operate at resonant frequency which results in low or no parasitic vibration modes, i.e. no jitter in the scanned line.

\section{Better dynamic response:}

Can be designed for the best dynamic response of the mirror (by applying and testing different actuator voltages and frequencies). Hence, less distortion again.

\section{Different modes operation:}

Fast and broadband capability allows different modes of operation, e.g. constant velocity line scanning and point to point step scanning.

\section{Low power:}

Small size and operating at resonant frequency results in low actuator voltage and power consumption.

\section{High efficiency:}

Mirrors can be coated with different materials to ensure high reflectivity. These materials can be also selected to compensate for the generated heat.

\section{Low cost:}

It can be built by using silicon-on-insulator (SOI) process which is a low-cost technology. 


\section{Brightness:}

Laser light in mirror scanner can be focused to a very small beam. Furthermore, the laser beam will not spread in space. This results in more brightness of the scanned line.

One drawback of micromirror laser line generator which limits its application is its low power characteristic. For example, it is almost impossible to use micro mirror to make a laser engraver and cutter for woods and metals. A laser cutter for wood requires about 30 to $40 \mathrm{~W}$ power. Also, a laser paper cutter should be of hundreds of milliwatts. The low power characteristic of mirror laser line generator stems from the damage threshold of the mirror.

\subsection{Applications of Micromirror Laser Line Generator}

Depending on the beneficial characteristics of micromirror laser line generator, it has been employed in many applications and has a good potential to be used in some other applications.

Some 1-Dimensional applications of micromirror laser line generator are alignment, identification (e.g. industrial inspection) and CD reader. Figures 3-7 to 3-9 illustrate these applications.

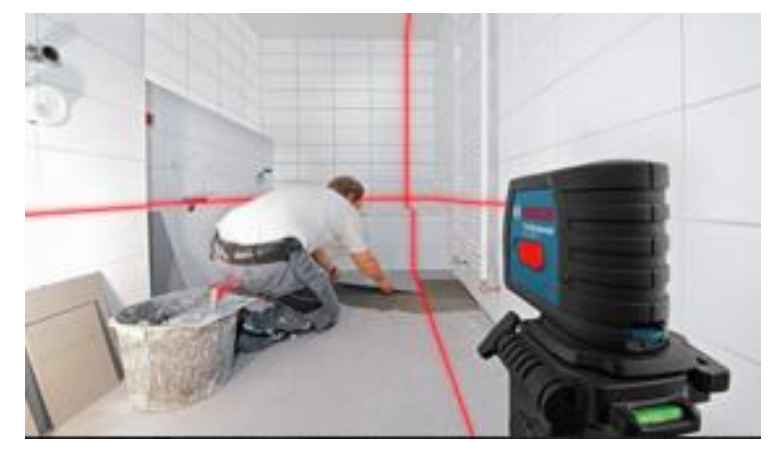

Figure 3-7. Laser line for perfect alignment.

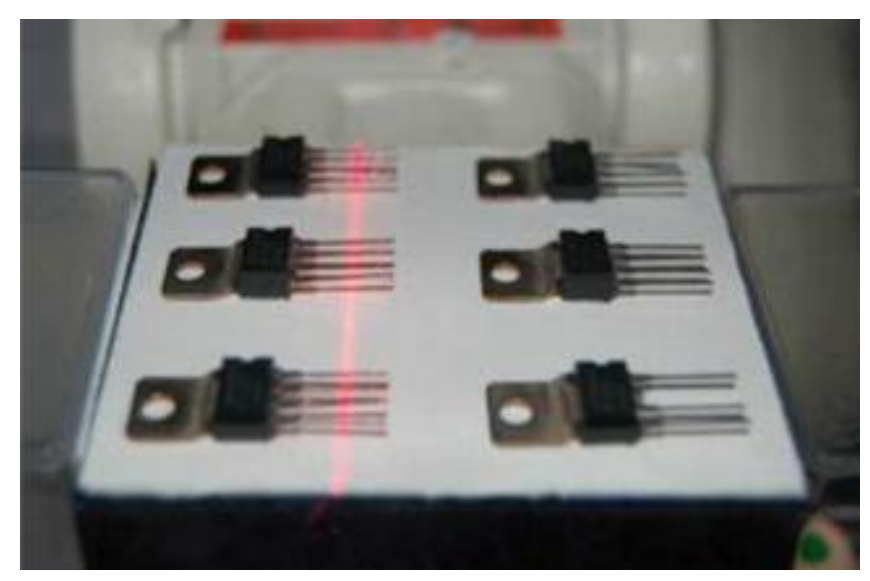


Figure 3-8. A laser line for leads inspection.

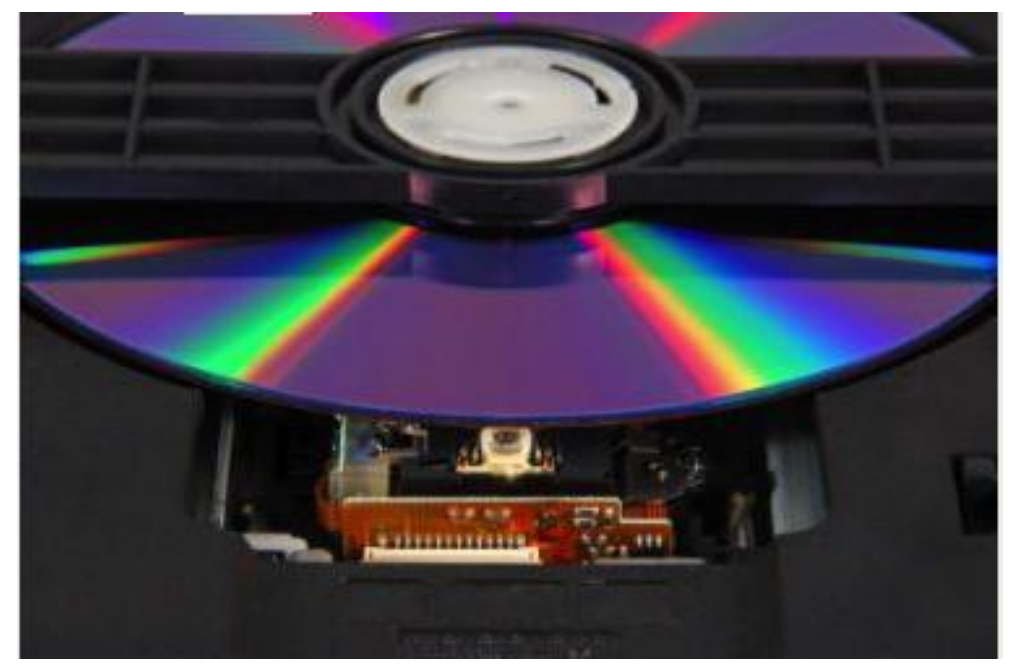

Figure 3-9. CD Reader.

Due to their low power consumption and small size, micromirror based laser line generators may be employed in barcode readers. Barcode readers scan a laser on a 1- Dimensional code (Figure 3-10) and detects the scattered light.

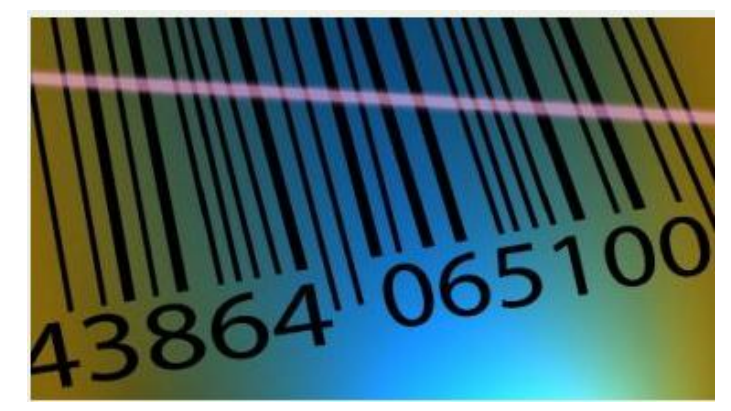

Figure 3-10. Barcode Scanner.

The main competitor of mirror line scanner in this application and some other imaging applications are linear imagers. A linear imager acts like a digital camera; it can read one or two dimentional codes. Reference [39] compares the characteristics of linear imaging and laser scanning technology (see Table 3-2) and shows that the micromirror laser line scanner has longer scan range and larger extended scan range. Moreover, it can result in more brightness of the scanned line for the same laser power and it has more capability in target detection. 
Table 3-2.Comparison of linear imaging and laser scanning technology [39].

\begin{tabular}{|c|c|}
\hline Application & Laser Scanner \\
\hline Scanning distance less than 18 inches $(45 \mathrm{~cm})$ & $\checkmark$ \\
\hline Scanning distance up to 35 feet (11 meters) & $\checkmark$ \\
\hline Higher bar code densities & Requires special scan engine \\
\hline Poor quality/damaged bar codes & $\checkmark$ \\
\hline Over-laminated bar codes & $\checkmark$ \\
\hline Linear bar code labels & $\checkmark$ \\
\hline 2D stacked bar codes - PDF 417 , Code 49 (requires special software) & Requires special scan engine \\
\hline Matrix codes (Datamatrix, QR code) & (see "Other Scanning Technologies") \\
\hline Reliability & \\
\hline Scan rate: $30-50$ scans per second & $\checkmark$ \\
\hline Scan rate: $200-800$ scans per second & Fixed position only \\
\hline Very bright spotting and scanning beam & $\sqrt{ }$ \\
\hline Fast scanning in fixed positions & $\sqrt{ }$ \\
\hline Retail Price (with cable): US\$150 - \$700 & $\checkmark$ \\
\hline Retail Price (with cable): US\$500 - \$2000 & $\checkmark$ \\
\hline
\end{tabular}

Micromirror laser line generator can be effectively used in a laser printer. The basic structure of a laser printer includes a rotating polygonal mirror which directs the light beam through a system of lenses and mirrors onto a photoreceptor drum (Figure 3.11). Laser printing is an application that requires high resolution and speed at low cost. Typically, $\theta-D$ product for this application should be about $60^{\circ} \mathrm{mm}$ that is hard to achieve. Moreover, the air damping must be controlled for reliable operation of the polygonal mirror, which in turn calls for a 
strong actuation. This problem can be solved by using a vacuum package but it increases the cost. Micromirror based laser line generator is best fitted to this application because its small size reduces damping and deformation. Moreover, it can oscillate at resonant frequency if it is designed for minimum damping. In a resonant scanner the mirror can have large angular deflection at a relatively high frequency with a small actuation.

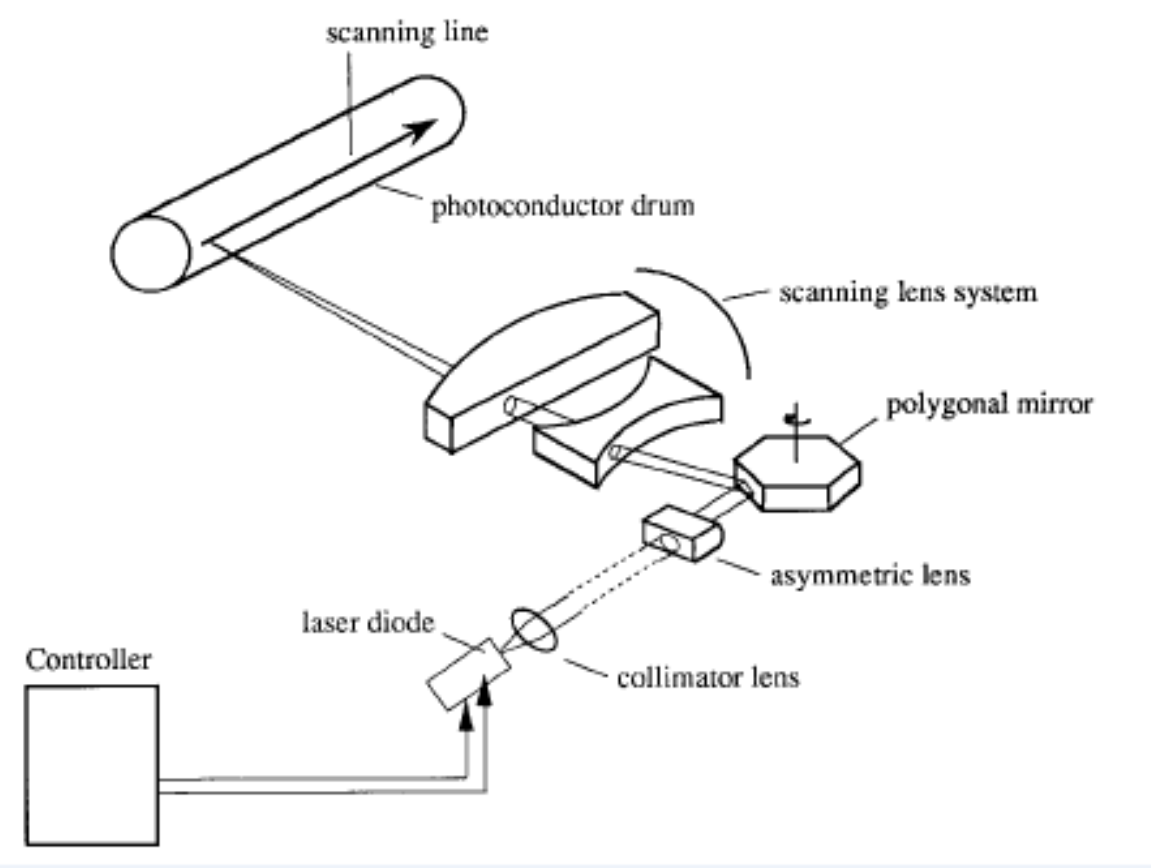

Figure 3-11. A typical structure of a laser printer [40].

Micromirror laser line generator has some good characteristics to be used in laser Doppler vibrometry and multi-touch screens. A conventional laser Doppler vibrometer has a relatively complex structure. Micromirror laser line generator is a proper candidate for this application because of its small size and low cost.

Micromirror laser line generator may be employed in multi-touch screen. In multi-touch screen, a light from a single or multiple lasers shine just above a surface. The laser plane or light is about $1 \mathrm{~mm}$ thick and positioned as close to the touch surface as possible. When a finger or object hits the light plane, the object lights up and is seen by camera below the surface. Alternatively, the finger may make a shadow which is again detected by sensors. An image is displayed by a projector or a computer. In multi-touch screen, usually 2 or 4 line generating lenses are placed in each corner of the surface. Mirror laser line generator can be used in this application for its small size, low power and uniform line. It may be possible to use fewer micromirror line generators because of its variable fan angle.

LADAR (LAser Detection And Ranging), which is equivalent to a laser RADAR, is another application of micromirror laser scanner. It can offer more advantages over other methods of 
target detection and analysis [38]. It uses a shorter wavelength than microwave radar and has high angular resolution. Micromirror laser line generator may be also used in spectrometers. Spectrometer is used in spectroscopy for producing spectral lines and measuring wavelength and intensities.

Micromirror laser line generator has many medical applications which are based on 2D/3D scanning, e.g. Optical Coherence Tomography(OCT). 


\section{Chapter 4 Designing the Laser Line Generator by Zemax}

In this chapter, a micromirror - based laser line generator is designed using Zemax OpticStudio 15 [41]. Zemax is a standard software which can be used for optical systems design, analysis, and optimization.

Based on the basic structure of Figure 3-5, the designated configuration for the micromirror laser line generator is shown in Figure 4-1. This figure is generated by Zemax software. The convex lens of Figure 3-5 has been omitted because the laser source generates a collimated beam. The SOI micromirror that is used in this design has a finite curvature. Thus a biconcave lens is used to amplify the scanning angle and compensate for the convergence of the laser beam reflected by the mirror. A reflective mirror is also used to direct the beam to the desired direction. Note that the selected laser source does not include a convex lens, i.e. it generates a fixed laser beam.

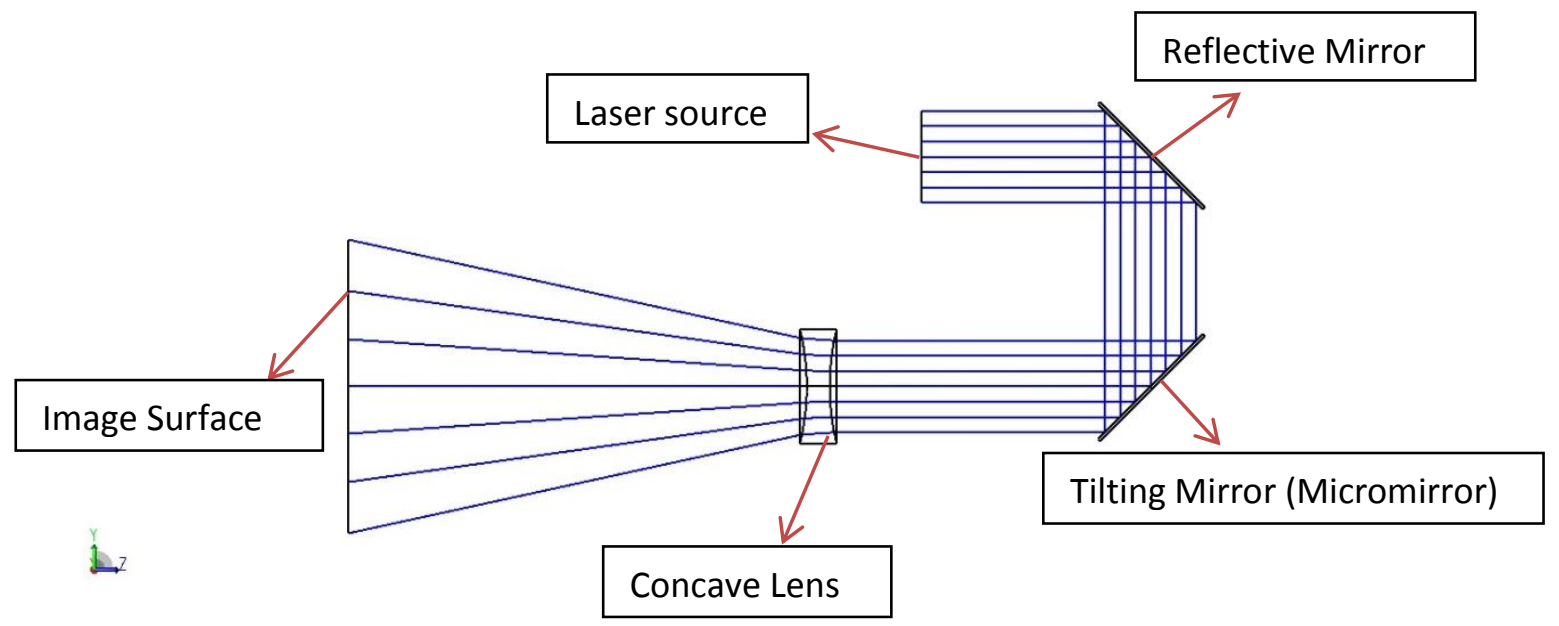

Figure 4-1. Designated Configuration.

To design the laser line generator, the following steps are taken in the software Zemax :

- Defining the components specifications and system parameters.

- Defining the designated configuration.

- Defining the micromirror operation and tilting angle.

In this study, reaching to the minimum spot size for the maximum possible image length is our target. One of the parameters that affect spot size is the distance between lens and 
micromirror. For achieving the best spot size, one can either change this distance manually or automatically by using the Optimization ability of the software.

The specifications of Micromirror, Laser and Lens are shown in the following Tables4.1 to 4.3.

Table 4-1. Micromirror specifications.

\begin{tabular}{|c|c|c|c|}
\hline & Dimension & Radius of Curvature & Mechanical Angle \\
\hline Micromirror & $1.5 \times 1.5 \mathrm{~mm}$ & $9.52 \mathrm{~cm}$ & 0 to 10 degree \\
\hline
\end{tabular}

Table 4-2. Laser specifications

\begin{tabular}{|c|c|c|c|}
\hline & Beam size & Wavelength & power \\
\hline Laser & $1 \mathrm{~mm}$ & $635 \mathrm{~nm}$ & $1 \mathrm{mw}$ \\
\hline
\end{tabular}

Table 4-3. Lens specifications.

\begin{tabular}{|c|c|c|c|c|}
\hline & Type & Diameter & Edge Thickness & Focal Length \\
\hline Lens & Double Concave lens & $12 \mathrm{~mm}$ & $4 \mathrm{~mm}$ & $-24 \mathrm{~mm}$ \\
\hline
\end{tabular}

By using these specifications and doing the pervious steps with the lens in $29 \mathrm{~mm}$ from the micromirror, the following results are achieved. Figure 4-2 shows the general view of the system and the beam trace for the tilting angle of \pm 2.35 degrees.

Remark: The rotation angle of micromirror is found by the measurement test and the distance between lens and micromirror is obtained by Zemax, as it will be explained in Section 5.2.

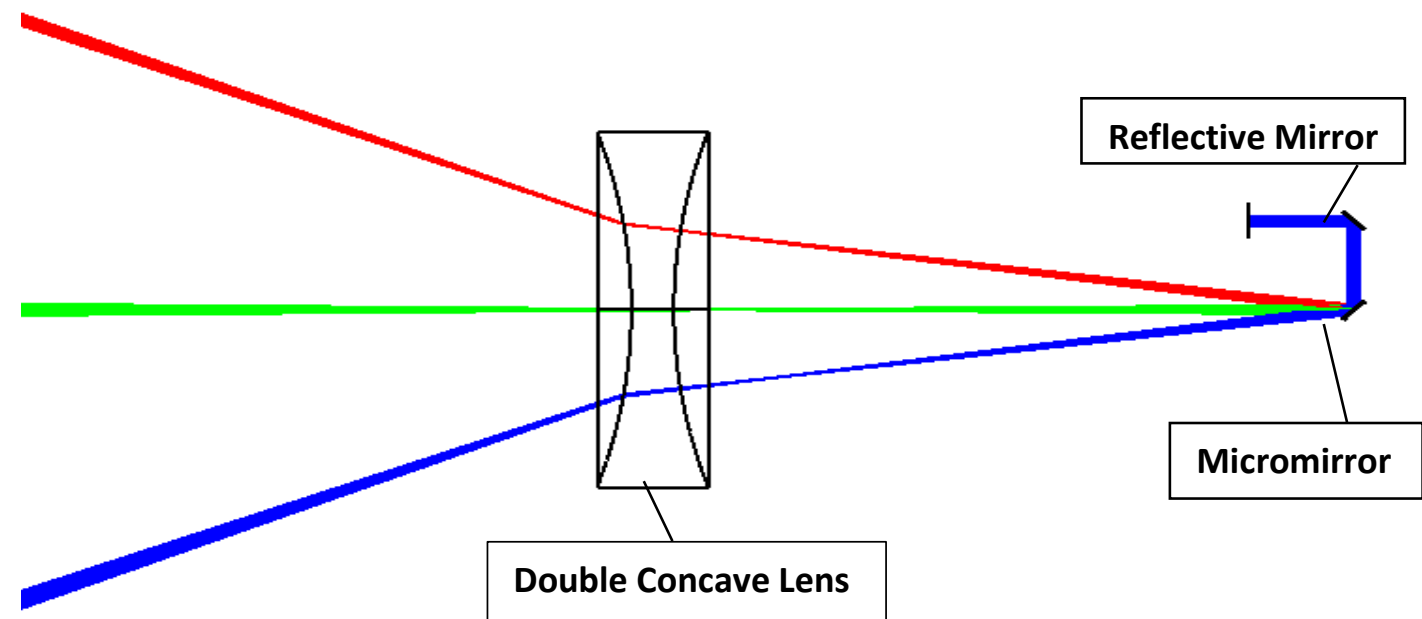

Figure 4-2. 2D view and beam trace 
Figure 4-3 shows the spot diagram at the image distance of $0.5 \mathrm{~m}$ for zero degree of micromirror tilting angle. Figure 4-4 illustrates the footprint (image length) of the scanner at the same distance.

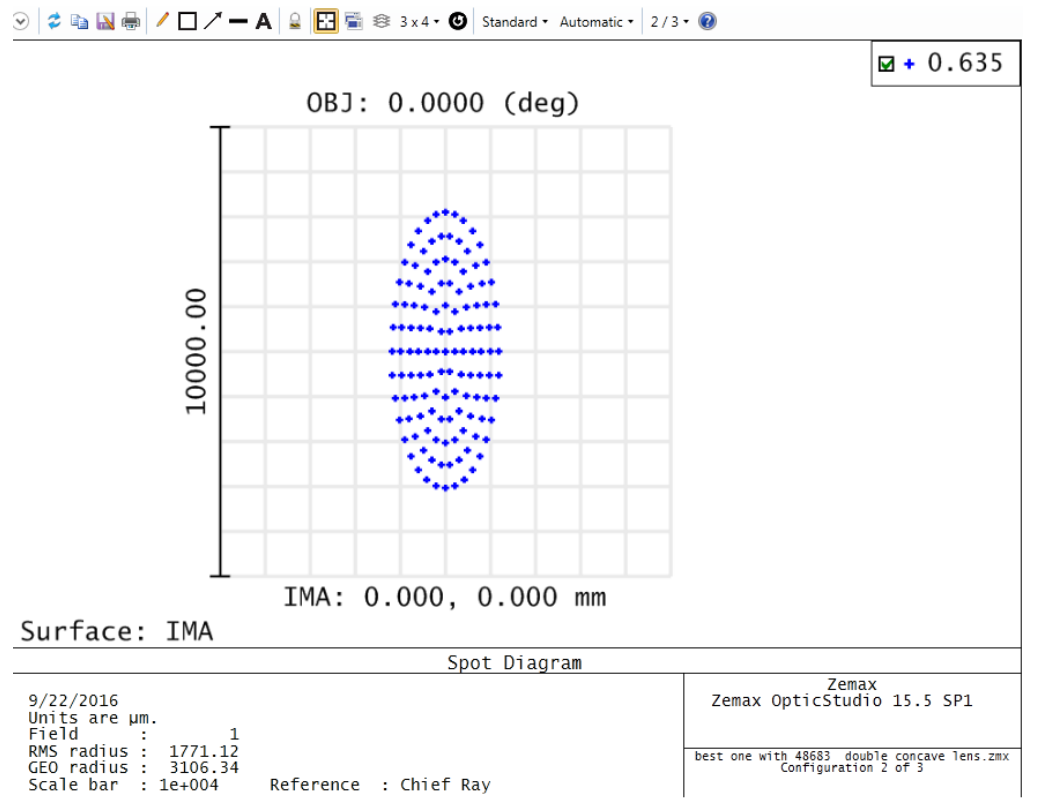

Figure 4-3. Spot Diagram ( $0.5 \mathrm{~m})$.

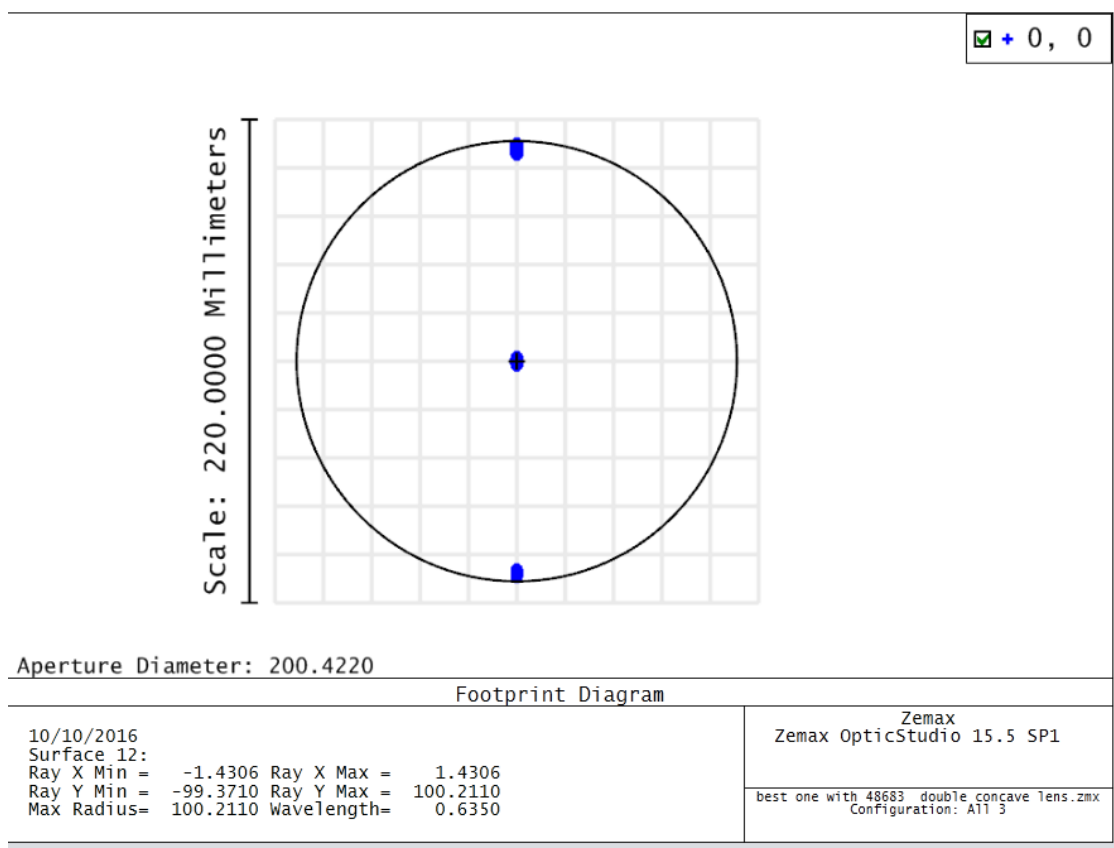

Figure 4-4.Footprint of the scanner ( $0.5 \mathrm{~m})$.

As it is seen from the above figures, the spot size and footprint of the scanner at the image distance of $0.5 \mathrm{~m}$ are $6.2 \mathrm{~mm} \times 2.3 \mathrm{~mm}$ and $200.4 \mathrm{~mm}$, respectively. Zemax software have been 
also used to simulate the spot sizes and footprints for image distances of 1 and 2 meters which have been shown in Figures 4-5 to 4-8 and tabulated in Table 4-4. As seen from Table $4-4$, the spot size and footprint is directly proportional to the image distance.

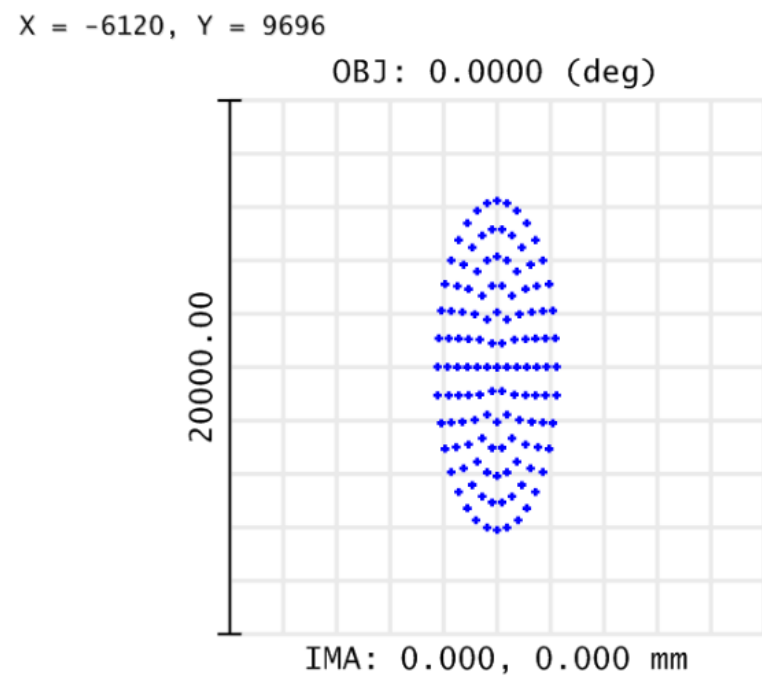

$\square+0.635$

Surface: IMA

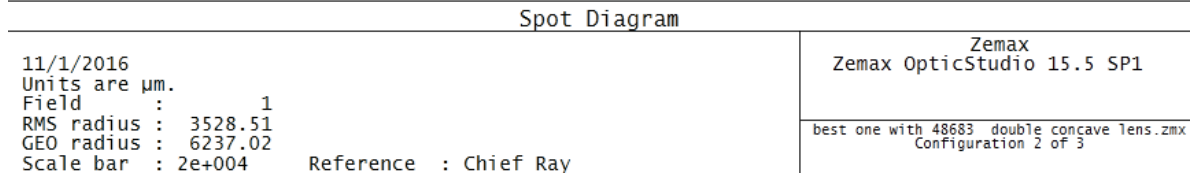

Figure 4-5. Spot Diagram ( $1 \mathrm{~m}$ ).

$\square+0,0$

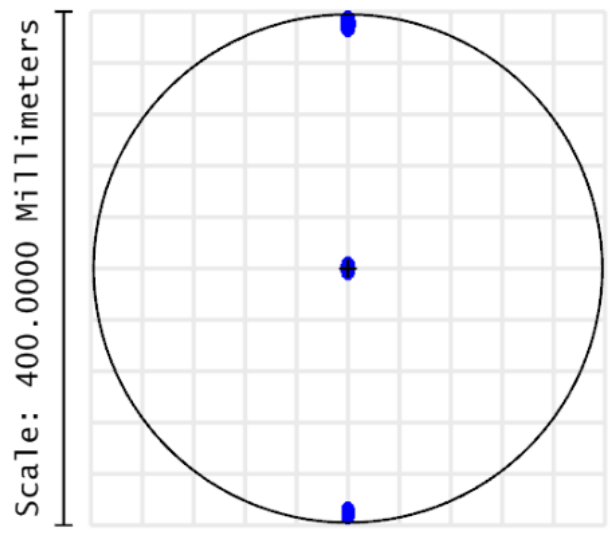

Aperture Diameter: 395.7828

Footprint Diagram

$11 / 1 / 2016$
Surface 12 :

Ray $X$ Min $=-2.7156$ Ray $X$ Max $=2.7156$

Ray Y Min $=-196.2330$ Ray $Y$ MaX $=$
Max Radius $=197.8914$ Wavelength $=$
0.6350

Zemax OpticStudio 15.5 SP1

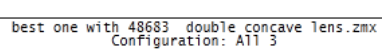

Figure 4-6. Footprint of the scanner ( $1 \mathrm{~m})$. 
OBJ : 0.0000 (deg)

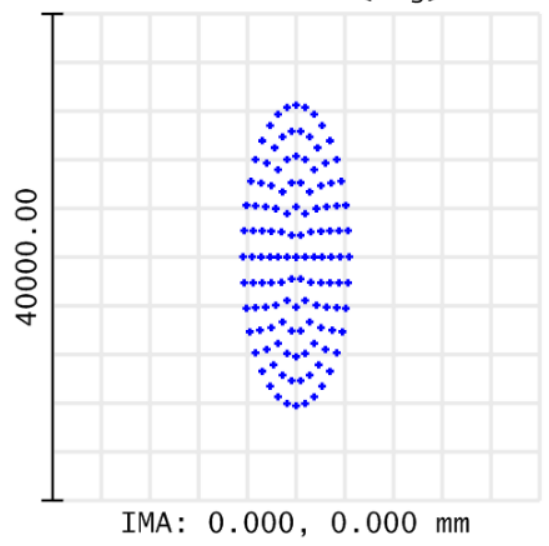

Surface: IMA

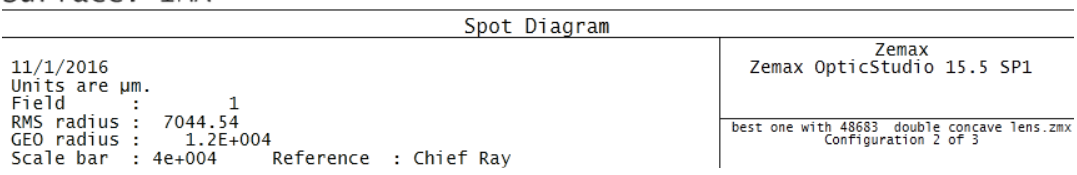

Figure 4-7. Spot Diagram ( $2 \mathrm{~m}$ ).

$X=-80.58, Y=-327.9$

$\square+0,0$

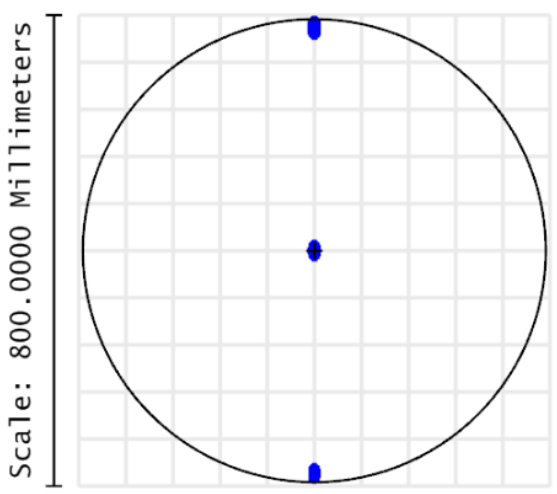

Aperture Diameter: 786.5045

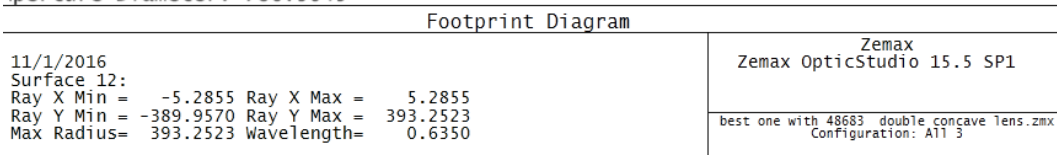

Figure 4-8. Footprint of the scanner ( $2 \mathrm{~m}$ ).

Table 4-4. Spot sizes and footprints.

\begin{tabular}{|c|c|c|c|}
\hline Distance & $500 \mathrm{~mm}$ & $1000 \mathrm{~mm}$ & $2000 \mathrm{~mm}$ \\
\hline Spot Size & $6.2 \mathrm{~mm} * 2.3 \mathrm{~mm}$ & $12.4 * 4.4 \mathrm{~mm}$ & $24 \mathrm{~mm} * 8.6 \mathrm{~mm}$ \\
\hline Scan Length & $200.4 \mathrm{~mm}$ & $395.7 \mathrm{~mm}$ & $786.5 \mathrm{~mm}$ \\
\hline
\end{tabular}




\section{Chapter 5 Shell Design and Test Results}

In this chapter, following a brief description about the designed shell for the micromirror based laser line generator, the function of its prototype is compared with the Zemax results.

\subsection{Shell Design}

Based on the configuration obtained by the Zemax software, a shell is designed to hold all mechanical, optical and electrical parts of the laser line generator. This is performed by the software "Solidworks". Solidworks is a solid modeling computer-aided design (CAD) and computer-aided engineering (CAE) program.

Considering the elements specifications, the elements supports and overall layout are designed by Solidworks software which are shown in Figures5-1 to 5-6.

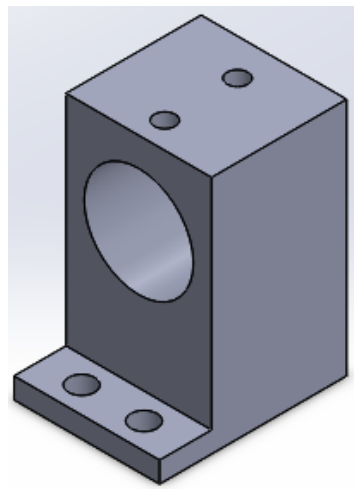

Figure 5-1. Laser support.

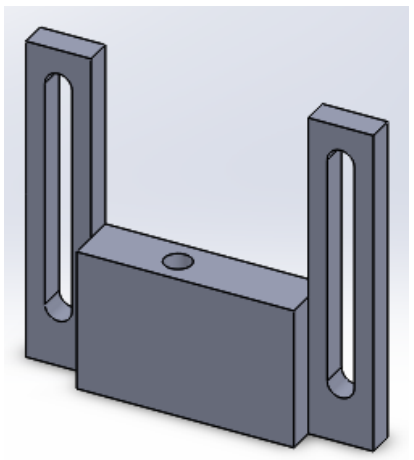

Figure 5-3. Tilting mirror support.

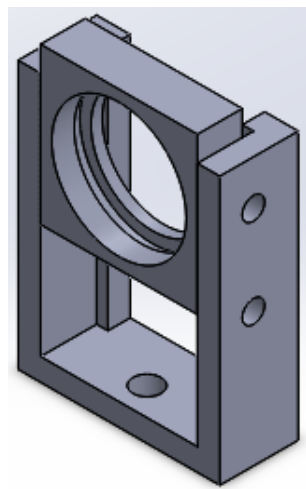

Figure 5-2. Lens support.

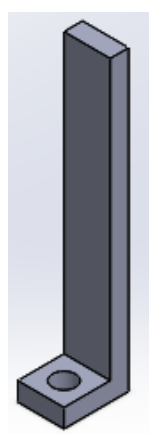

Figure 5-4. Mirror support. 


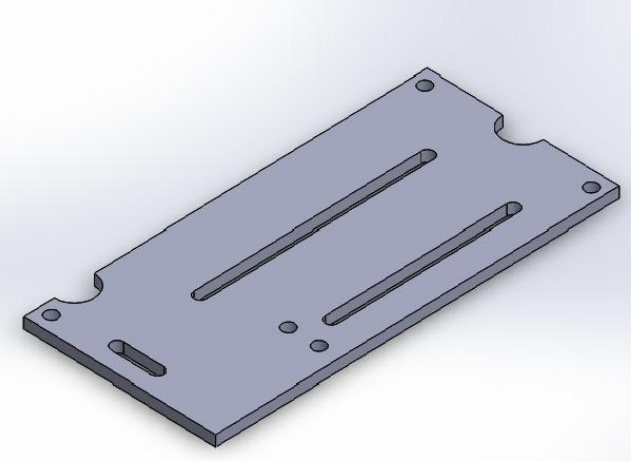

Figure 5-5.Base Plate.

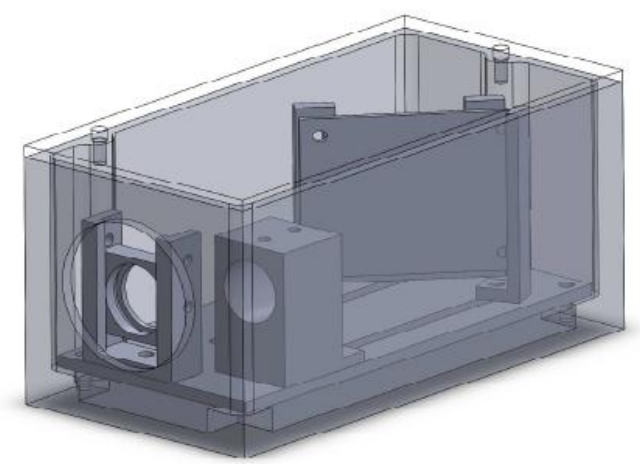

Figure 5-6. Overall layout.

Figure 5-7 illustrates the laser beam trace when all the components are put in their places.

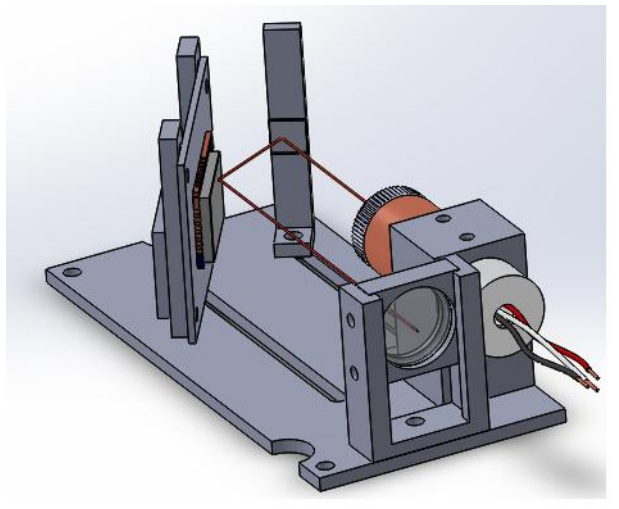

Figure 5-7. The laser beam trace.

\subsection{Test Results}

Figurer 5-8 shows the measurement set up for specifying the rotation angle of micromirror. A laser beam is projected on the mirror and the length of the scanning line is measured. A grid paper is used as the image screen. By performing a test without the lens, the optical scanning (mechanical) angle of the micromirror can be measured. 


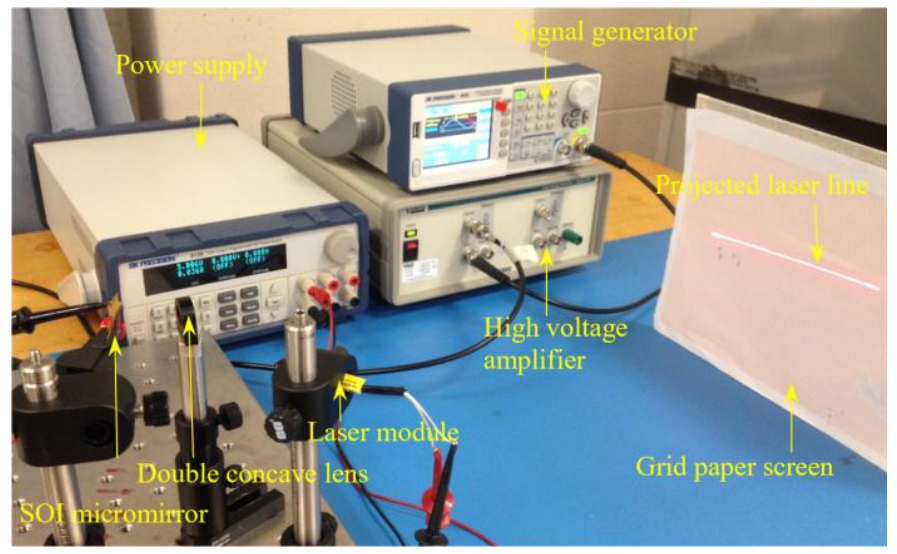

Figure 5-8. Measurement set up.

To achieve the maximum rotation angle of micromirror, it was actuated with a $100 \mathrm{~V}$ sinusoidal voltage at the resonant frequency $(940 \mathrm{~Hz})$. The maximum length of the image line without concave lens was measured as $82 \mathrm{~mm}$ at a distance of $0.5 \mathrm{~m}$ (Figure 5-9).

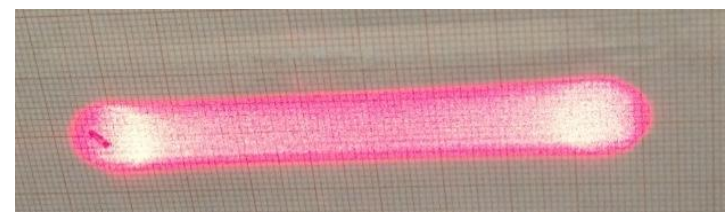

Figure 5-9. Scanned line.

Figure 5-10 illustrates how the rotation angle was calculated, where $\mathrm{D}$ is the distance between micromirror and screen and $\mathrm{L}$ is the length of scanned line.

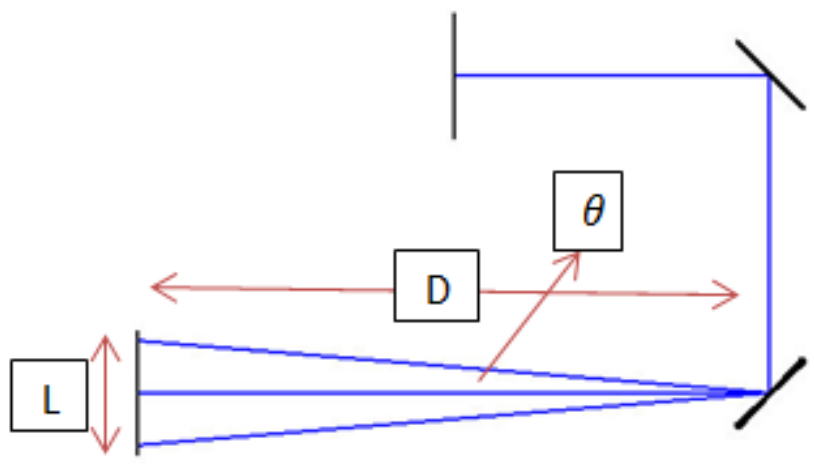

Figure 5-10. Optical angle of micromirror.

As it is seen from this figure, the optical angle of scanning micromirror without the concave lens is given by the following relation.

$$
\theta=\operatorname{Arctan} \frac{L}{2 D}
$$


By using the above relation for $\mathrm{L}=82 \mathrm{~mm}$ and $\mathrm{D}=500 \mathrm{~mm}$, the optical angle without the concave lens can be calculated as 9.4 degrees. Mechanical angle is half of optical angle, so the rotation angle of micromirror is \pm 2.35 degrees.

In the next step, the Zimax software was used to optimize the distance between the micromirror and concave lens. The optimization criterion was to minimize the spot size for maximum possible scanning length. The best result was obtained when the distance between micromirror and lens was set at $29 \mathrm{~mm}$ where the length of scanning line was $200.4 \mathrm{~mm}$ (Figure 4-4).

Assembled laser line generator is shown in Figure 5-11(a) which was enclosed in a box (88mm x 42mm x 46mm) as shown in Figure 5-11(b).

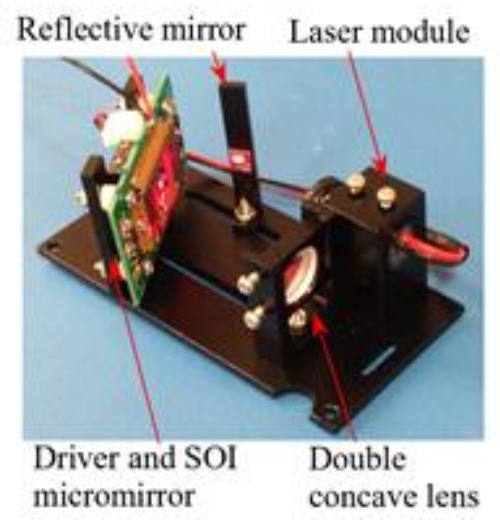

(a)

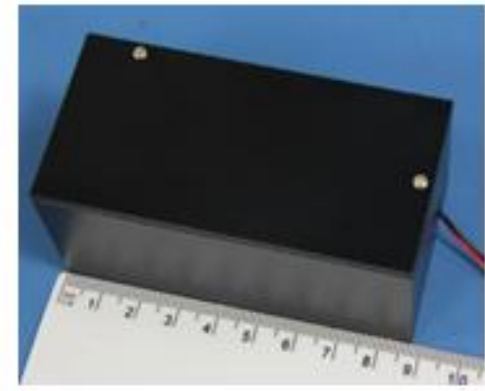

(b)

Figure 5-11. (a) Assembled (b) packed laser line generator.

To verify the operation of the prototype model, its micromirror was actuated for the same mechanical angle. The measured length of scanned line was equal to $200 \mathrm{~mm}$ at distance $0.5 \mathrm{~m}$ which was very close to the Zemax result $(200.4 \mathrm{~mm})$. Finally, based on the achieved results, it was concluded that the concave lens amplified the fan angle of micromirror by a factor of 2.4.This value is close enough to its theoretical value. The angle amplification factor of concave lens $\theta_{o} / \theta_{i}$ can be determined from Equation 3-9 which is repeated here:

$$
\tan \theta_{o}=\left(1+\frac{p}{f_{n}}\right) \cdot \tan \theta_{i}
$$


$\theta_{o}$ and $\theta_{i}$ are the angles of laser beam before and after the concave lens, $\mathrm{P}$ is the distance between micromirror and lens, and $f_{n}$ is the focal length of the concave lens. Based on the Equation (5-11), it can be shown that the lens amplification factor is about 2.2.

An advantage of micromirror based laser generator compared with conventional laser line generator is its variable fan angle. Conventional laser line generators have fixed fan angle and, consequently, when the working distance increase, their projected laser lines become too long to have enough power density/ brightness. On the other hand, micromirror based laser line generators have adjustable fan angles such that they can retain the power density unchanged for various working distances. This difference is demonstrated in Figure 5-12.

The resolution of the designed micromirror based laser generator can be determined from the following relation which was also given in section 3.2.

$$
N=\frac{\theta \mathrm{D}}{a \lambda}
$$

The shaping factor " $a$ " for a square mirror is equal to 1 . For $\theta=9.4$, the resolution is calculated to be about 380 pixels.

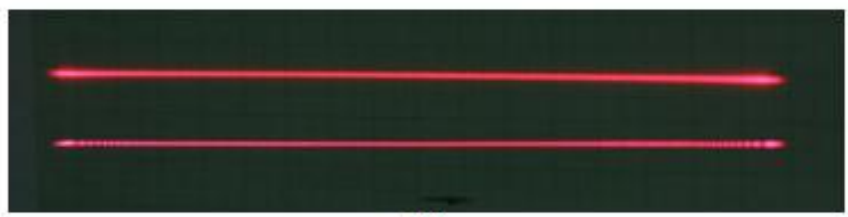

(a)

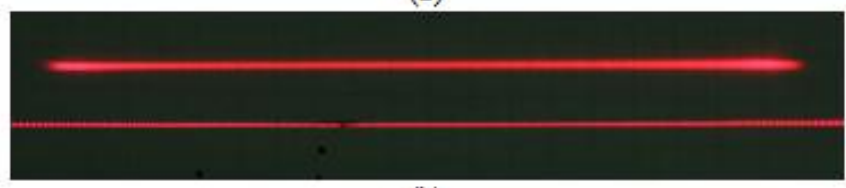

(b)

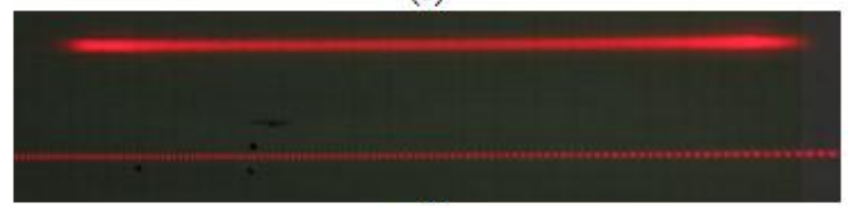

(c)

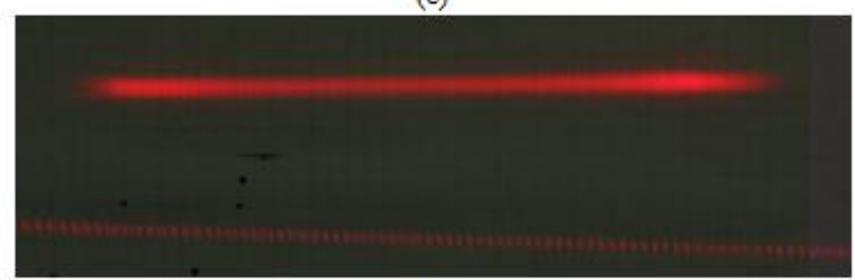

(d)

Figure 5-12.Brightness comparison of micromirror based laser line generator and conventional laser line generator at distances (a) $5 \mathrm{~m}$, (b) $1 \mathrm{~m}$, (c) $2 \mathrm{~m}$, (d) $3 \mathrm{~m}$. 


\section{Chapter 6 Conclusions}

SOI micromirrors which can be resonantly driven by different actuation methods offer several advantages like high scan frequency, very small size and potential for low manufacturing cost. As the main goal of this project, a SOI micromirror based laser line generator has been designed, implemented, tested and compared with a conventional laser line generator.

As it has been mentioned in Chapter 3, a micromirror based laser line generator is constructed from a laser module, a tunable micromirror and some lenses to shape the laser beam. The types and number of lenses used depend mainly on the laser source and mirror curvature. In fact, for a collimated laser beam and a flat micromirror, there is no need of lens.

In Chapter 4, Zemax software has been used to design and simulate the micromirror based laser line generator. Zemax is an standard industrial software which can be used for optical systems design and simulation. The designated configuration for the laser line generator consisted of a laser module, a reflected mirror, a tilting micromirror and a double concave lens. The reflective mirror has been used to direct the laser beam to the desired direction. The double concave lens has been placed between the tilting mirror and the image plane to compensate for the micromirror curvature and to amplify the micromirror fan angle.

The distance between the concave lens and micromirror plays an important role in forming the spot size and line length for a fixed image distance. To achieve the best result (small spot size and large line length), this distance can be changed manually in the software or automatically by using the optimization ability of software. Based on the Zemax simulation results, a distance of $29 \mathrm{~mm}$ between lens and micromirror together with a fan angle of 4.7 degrees have resulted in an image length of $200.4 \mathrm{~mm}$ at the image distance of $0.5 \mathrm{~m}$. The tilting angle of micromirror corresponds to the micromirror resonant frequency of $9400 \mathrm{~Hz}$ at $100 \mathrm{~V}$ actuating voltage.

To fabricate the designed scanner, engineering software SolidWorks has been used to design a shell to hold all mechanical, optical and electrical parts. The assembled laser line generator has been shown in Chapter 5. The assembled generator has been also tested and compared with a conventional laser line generator. As it has been shown in Chapter 5, the micromirror based laser line generator can keep the image line length and brightness unchanged at different image distances; which is not possible with conventional laser line generators. This benefit of micromirror based laser line generator is due to its adjustable fan angle. In fact, 
micromirror based laser line generators can offer more advantages like high scanning rate, small size and low distortion which have been mentioned in Chapter 3.

A disadvantage of micromirror laser line generator is that the tilting mirror may lose its alignment and change its scanning performance.

Micromirror based laser line generator has been or can be employed in many applications like alignment, barcode reading, LIDER, etc. It has also good potential to be used in some other applications. Doppler vibrometer and large multi-touch screen may be two applications which can be investigated in future works.

Finally, the designed package for the micromirror based laser line generator has not been optimized for its size. This optimization, which is necessary for high volume production, should be done if required. 


\section{References:}

[1] C.Y.Wang, Q.C.Tan and R.H.Guo, "Design and optimization of linear laser beam," Lasers in Eng.,Vol. 27, pp. 373-381(2014).

[2] http://www.laserlineoptics.com/powell_primer.html (2016 11)

[3] K.E Petersen, " Silicon torsional scanning mirror," IBM J.of Res. Develop. Vol. 24, No.

5,pp. 631-637, (1980).

[4] http://spie.org/publications/fundamentals-of-photonics-modules (2016 11)

[5] O.Solgaard, et el., "Optical MEMS: From micromirrors to complex systems," J. of Micromechanical systems, Vol. 23,No. 3,pp.517-538(2014).

[6] H. Schenk et al., "Design and modeling of large deflection micromechanical 1D and 2D scanning mirrors," Proc. SPIE, 4178, pp. 116-125(2000).

[7] R. A. Conant et al., "Robustness and reliability of micromachined scanning mirrors," Proc. IEEE-LEOS Optical MEMS(1999).

[8] V. A. Aksyuk et al., "Micromirror array technology for large optical crossconnects," Proc. SPIE, 4178, pp. 320-324(2000).

[9] T. J. Kaiser et al, "Silicon nitride biaxial pointing mirror with stiffening ribs, " Proc. SPIE 4561, pp. 276-282 (2001).

[10] Schenk et al., "A new driving principle for micromechanical torsional actuators, "Micro Electro Mechanical Systems, ASME, pp. 333-338(1999).

[11] K. Torashina et al.,"A micro scanner with low power consumption using double coil layers,” Proc. IEEE-LEOS Optical MEMS, pp. 184-184(2004).

[12] F.Filhol et al., "Piezoelectric micromirrors for fast optical scanning with large angular deflection,” Proc. IEEE-LEOS Optical MEMS, pp. 192-193(2005).

[13] G. Lammel et al., "Low cost microspectrometer,” Proc. SPIE 4178, pp. 288-295(2000).

[14] A.Cowen et el., SOIMUMPs Design Handbook, MEMSCAP Inc., (2011).

[15] http://www.microcletech.com

[16] H.Urey,et al., "Optical performance requirements for MEMs-scanner based microdisplays,” Proc.SPIE, Vol. 4178, pp.176-185(2000).

[17] A. Wolter, et el., "Applications and requirements for MEMs scanner mirrors," Proceedings of SPIE, Vol. 5719, pp. 65-75(2005)

[18] L.Beiser and R.Barry Johnson, Handbook of Optics, 3rd Ed., Vol. 1, Chapter 19.

[19] M. Born and L. Wolf, Principle of Optics, $6^{\text {th }}$ ed., Pergamon (1980) 
[20] P.J. Brosens, "Dynamic mirror distorsions in optical scanning," Applied Optics, Vol. 11,pp. 2988-2989(1972).

[21] H. Urey, et al., "Scanner design and resolution tradeoffs for miniaturized scanning displays," Proc. SPIE, Vol. 3636, pp. 60-68(1999).

[22] M.H. Kiang, et el., "Surface-micromachined electrostatic-comb driven scanning micromirrors," (1996)

[23] M.H. Kiang, et el., "Electrostatic combdrive-actuated micromirrors for laser-beam scanning and positioning," J. of Micro Electro Eechanical Systems,Vol.7, No.1, pp. 2736(1998)

[24] K. H. Koh and C. Lee, “A low power 2-D raster scanning MEMS mirror driven by hybrid electrothermal and electromagnetic actuation mechanism," Proc. IEEE Optical MEMS Nanophoton, pp. 236-237(2012).

[25] L. Li et al., “ A symmetric hybrid MEMS scanner with electrothermal and electrostatic actuators," Proc. IEEE Optical MEMS Nanophoton, pp. 163-164,(2011).

[26] S. T. S. Holmstrom, et al., "MEMS laserscanners; A review," J. of Microelectromechanical Systems, Vol.23, No. 2, pp.259-275(2014).

[27] D. W. Wine et al., "Perfomance of biaxial MEMS- based scanner for micro display applications,” Proc. SPIE 5604, pp. 218-219(2004).

[28] P.R. Patterson, et al., "Scanning micromirror: An overview," Proc. SPIE, Vol. 5604, pp. 195-207(2004).

[29] A. Selvakumar and K. Najafi, "Vertical comb array microactuators," IEEE J. Microelectromechanic Systema, Vol. 12, No. 4, pp. 440-449(2003).

[30] H. Urey, "MEMS scanners for display and imaging applications," Proc. SPIE 5606, pp.218-219(2004).

[31] J. Yan et al., "Magnetic actuation for MEMS scanners for retinal scanning displays," Proc. SPIE , 4985, pp. 115-120(2003).

[32] K. Uchino, “Piezoelectric actuators,” J. Electroceramics, Vol. 20, pp. 301-311(2008).

[33] J. A. Connally and S.B. Brown, "Slow crack in single crystal silicon," Science, New Series, Vol. 256, No. 5063, pp. 1537-1739(1992).

[34] A. Wolter et al., "Torsional stress, fatigue and fracture strength in silicon hinges of microscanning mirror,' Proc. SPIE 5343, pp.176-185(2000).

[35] C. L. Mulstein, "High-cycle fatigue of single-crystal silicon thin film," J. MEMS, Vol. 10, No. 4, pp.593-600(2001). 
[36] A. D. Yalcinkaya et al., "Two-axis electromagnetic microscanner for high resolution displays,' J. Micro Elecro Mechanical Systems, Vol.15, No. 4, pp. 786-794(2006).

[37] U. Baran et al., “ Resonant PZT MEMS scanner for high resolution displays,” J. Micro Eelectro Mechanical Systems, Vol. 21, No. 6, pp.1303-1310(2012).

[38] J.P. Siepmann and A. Rybaltowski," Integrable ultera-compact, high resolution, realtime MEMs LADAR for individual soldier," MILCOM (2005).

[39] www. intermec.com

[40] S. Nakatsuka, et al., "Resolution enhancement printing with a variable spot- size laser diode," Applied Optics, Vol. 36, No. 24, pp.5876-5880(1997).

[41] www.Zemax.com, Getting started with OpticStudio 15, May (2015). 